\title{
Strontium and oxygen isotope evidence for the origin and movement of cattle at Late Neolithic Durrington Walls, UK
}

\author{
Jane Evans ${ }^{1}\left([) \cdot\right.$ Mike Parker Pearson $^{2} \cdot$ Richard Madgwick $^{3} \cdot$ Hilary Sloane $^{1} \cdot$ Umberto Albarella $^{4}$
}

Received: 14 December 2018 / Accepted: 25 April 2019 / Published online: 6 June 2019

(C) The Author(s) 2019

\begin{abstract}
The geographic origins of livestock found at the Late Neolithic site of Durrington Walls (Wiltshire, UK) is explored using strontium $\left({ }^{87} \mathrm{Sr} /{ }^{86} \mathrm{Sr}\right)$ and oxygen $\left(\delta^{18} \mathrm{O}_{\text {carbvSMOW }}\right)$ isotope analysis of tooth enamel as an archive of lifetime movement. The analysis of 49 cattle is augmented with data for small numbers of animals from the contemporaneous monumental centres of West Kennet Palisade Enclosures (4), Stonehenge (1), and Marden (1). Unburnt human remains are scarce at these sites and the suite of biomolecular analyses that can be undertaken on cremated remains is limited. Therefore, these animals provide the best proxy for the origins of the people who raised them and give key information on livestock management. This builds on the Sr isotope analysis of 12 animals previously published from Durrington Walls and complements recent research on pig remains from the same sites, providing further evidence for the scale of human and animal movement and the catchment of these sites. The strontium isotope signatures from the animals' teeth range between values that are consistent with local chalkland grazing to radiogenic values typical of granites and older rock types. The oxygen isotope data, coupled with the strontium results, provide new geographic resolution and indicate that the majority of the animals come from southern and western areas of Britain.
\end{abstract}

Keywords Bos taurus $\cdot$ Strontium $\cdot$ Oxygen $\cdot$ Isotopes $\cdot$ Durrington Walls $\cdot$ Mobility $\cdot$ Neolithic

Electronic supplementary material The online version of this article (https://doi.org/10.1007/s12520-019-00849-w) contains supplementary material, which is available to authorized users.

Jane Evans

je@bgs.ac.uk

Mike Parker Pearson

m.parker-pearson@ucl.ac.uk

Richard Madgwick

madgwickrd3@cardiff.ac.uk

Hilary Sloane

hjs@bgs.ac.uk

Umberto Albarella

u.albarella@sheffield.ac.uk

1 National Environmental Isotope Facility, British Geological Survey, Keyworth NG12 5GG, UK

2 Institute of Archaeology, UCL, 31-34 Gordon Square, London WC1H 0PY, UK

3 School of History, Archaeology and Religion, Cardiff University, Cardiff CF10 3EU, UK

4 Department of Archaeology, University of Sheffield, Minalloy House, 10 - 16 Regent Street, Sheffield S1 3NJ, UK

\section{Background}

\section{Archaeological context and previous work}

During the Late Neolithic, Durrington Walls was the focus of feasting activities that included the slaughter and consumption of a substantial number of animals (Albarella and Serjeantson 2002; Richards and Thomas 1984). This was evidenced by the work carried out on the considerable quantity of faunal remains associated with the henge, its internal features, and extensive middens excavated in the 1960s (Wainwright and Longworth 1971) and, between 2004 and 2007, by the Stonehenge Riverside Project (Parker Pearson et al. 2011). Among the animals consumed at the site, pigs (Sus domesticus) were predominant, but the remains of cattle (Bos taurus) were also abundant in the zooarchaeological assemblage. Evidence from pig age-at-death analysis suggests that the intensity of feasting varied seasonally, with a peak during the winter months (Albarella and Payne 2005; Wright et al. 2014). The question of how, and from where, such largescale consumption was provisioned was one of the central research foci of the Feeding Stonehenge Project, funded by the Arts and Humanities Research Council (AHRC). 
A pilot study carried out in 2009-2010 hinted that the provisioning of livestock to the site was complex. Using strontium isotope analysis of cattle teeth, the research suggested that livestock found at the site were from diverse geographical origins. While some of the cattle were likely to be local, a proportion of the animals had ${ }^{87} \mathrm{Sr} /{ }^{86} \mathrm{Sr}$ values that were inconsistent with the Cretaceous chalk on which the site is located (Viner et al. 2010). These animals must have been raised away from Durrington Walls and transported to the site after their teeth had formed.

The aim of this paper is to provide detail to the story of cattle origins in the Late Neolithic. A larger dataset, the integration of strontium and oxygen isotope analysis, and the inclusion of a small number of teeth from other Late Neolithic sites (West Kennet Palisade Enclosures, Marden and Stonehenge) in the study area were all necessary to provide greater resolution (Fig. 1). This approach has the potential to determine the extent of cattle movement to Durrington Walls, the likely origins of the livestock that had been moved, and whether the movement of cattle was part of a wider phenomenon of livestock mobility.

\section{Tooth formation}

Tooth enamel does not undergo any remodelling and hence its isotope composition is fixed during the period of formation. This means that analytical results from cattle third molar enamel will provide a snapshot of the prevailing conditions during the period from approximately 9 to 30 months of a cattle's life (Balasse 2002). Unlike tooth enamel, both tooth dentine and bone are susceptible to diagenetic alteration due to their more porous and less crystalline structure (Trickett et al. 2003); hence, bone and dentine provide information about the burial environment rather than a lifetime signature. In addition, these tissues remodel throughout life so, even if they were not diagenetically affected, they would provide a blended isotope signal for the years before death that can be difficult to interpret.
Fig.1 A map showing the range of median ${ }^{87} \mathrm{Sr} /{ }^{86} \mathrm{Sr}$ values of domains across Britain with the position of the study area that includes Durrington Walls, Marden, Stonehenge and West Kennet sites, and Irthlingborough, Chillingham, and Durham locations.

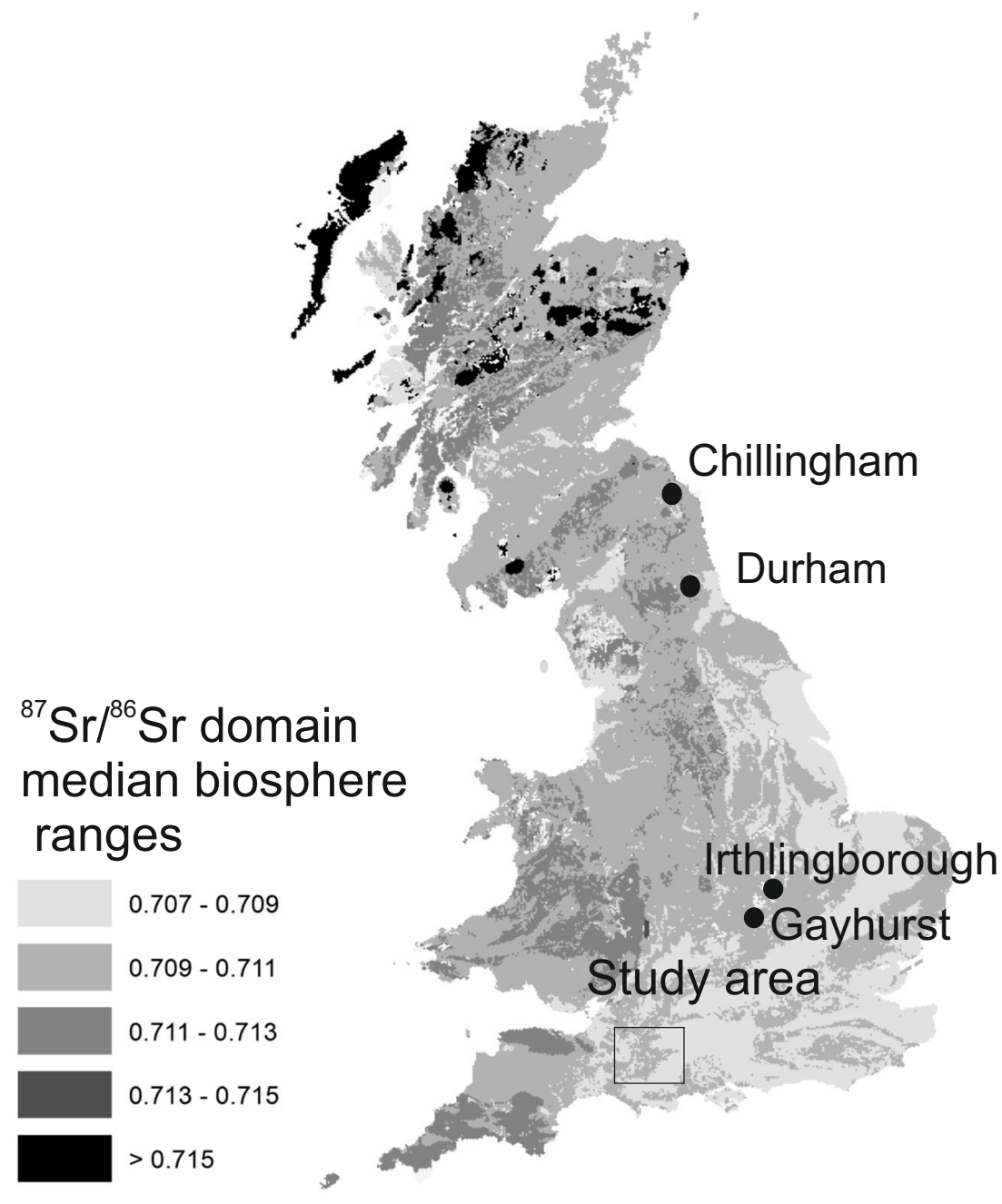




\section{Isotopes}

\section{Strontium $\left({ }^{87} \mathrm{Sr} /{ }^{86} \mathrm{Sr}\right)$ isotope analysis}

Strontium isotope analysis is an effective tool for identifying mobility in the past. It has been used successfully by researchers interested in tracking animal migration (Britton et al. 2009, 2011; Hoppe et al. 1999; Julien et al. 2012), for the study of trade networks (Madgwick et al. 2012, 2019a; Madgwick and Mulville 2015; Minniti et al. 2014; van der Jagt et al. 2012), and patterns of animal movement (Balasse et al. 2002; Bendrey et al. 2009; Bentley 2006; Bogaard et al. 2013; Evans et al. 2007; Sykes et al. 2006; Viner et al. 2010). The method is based on the principle that rubidium ${ }^{87} \mathrm{Rb}$ decays to ${ }^{87} \mathrm{Sr}$ over time, changing the ratio of ${ }^{87} \mathrm{Sr}$ to ${ }^{86} \mathrm{Sr}$. As a result, older rocks and $\mathrm{Rb}$-rich rocks have higher ratios of ${ }^{87} \mathrm{Sr} /{ }^{86} \mathrm{Sr}$, with the effect that biologically available ${ }^{87} \mathrm{Sr} /{ }^{86} \mathrm{Sr}$ varies spatially according to the age and chemistry of the underlying geology. The link between biologically available $\mathrm{Sr}$ and underlying geology is well documented (Ericson 1985), but other factors, such as the ${ }^{87} \mathrm{Sr} /{ }^{86} \mathrm{Sr}$ ratio in ground and river water, and in some cases sea-spray (Bentley 2006), may also contribute.

All the sites in this study are located on Cretaceous chalk, which has a relatively well-defined strontium isotope biosphere range of $0.7083 \pm 0.0006$ ( $1 \mathrm{SD}, n=85$; Evans et al. 2018). Dentine and bone samples from Durrington Walls give a similar result of $0.7086 \pm 0.0004$ ( $1 \mathrm{SD}, n=11$; Viner et al. 2010), which is to be expected for tissues that absorb the burial environment strontium isotopic signal. These data represent the predicted $\mathrm{Sr}$ isotope composition for tooth enamel of animals that graze on a chalk-founded terrain.

\section{Oxygen $\left(\delta^{18} 0\right)$ isotope analysis}

Oxygen isotope analysis has also been successfully applied to the investigation of animal mobility (Britton et al. 2009; Henton et al. 2010; Madgwick et al. 2019a; Towers et al. 2011, 2017). Environmental oxygen isotope ratios are dependent on the hydrological system and reflect the fractionation of ${ }^{18} \mathrm{O}$ compared with ${ }^{16} \mathrm{O}$. In general, precipitation is increasingly depleted in ${ }^{18} \mathrm{O}$ at high latitude (Bentley and Knipper 2005). In Britain, because our weather systems are predominantly from the Atlantic, the oxygen isotope zonation in Britain is predominantly west to east; the lowest values were recorded in the eastern Highlands of Scotland (Darling et al. 2003). Oxygen isotope ratios in tooth enamel have a linear relationship with water ingested during the period of tooth formation and can therefore provide information about the geographical origin of animals from archaeological sites. However, using calibrating equations to 'map' enamel values on to geographically determined water values introduces additional uncertainty onto the data, and their application is therefore questionable (Pollard et al. 2011). In this study, we have used data from animals from central and eastern England, which represent the British drinking water zone of -7 to $-8 \%$, as defined by Darling et al. (2003). These provide reference data against which we compare the Late Neolithic data. These comparative datasets come from the feral cattle from modern Chillingham Castle in Northumberland: (Towers et al. 2014, 2017) and Bronze Age cattle from Irthlingborough, Northamptonshire, and Gayhurst, Buckinghamshire (Towers et al. 2011).

\section{Carbon $\left(\delta^{13} \mathrm{C}\right)$ isotope analysis}

Carbon isotopes in tooth enamel record the $\delta^{13} \mathrm{C}$ of plants consumed by cattle. These data are of limited use for addressing the core aims of this research, centering on origins and movement, but are presented here to augment the oxygen isotope data and to explore varied husbandry strategies. Plants are divided into two main groups based on their photosynthetic pathways: C3 and C4 (Schwartz and Shoeninger 1991), and these two groups have substantially different $\delta^{13} \mathrm{C}$ isotope ranges. However, $\mathrm{C} 4$ plants, which are common in more arid environments, are rare in the indigenous flora of the British Isles and northern Europe. Therefore, British studies are restricted to variations in terrestrial $\mathrm{C} 3$ plants, and the transmission of the carbon $\delta^{13} \mathrm{C}$ composition to the animals that graze on them. This range has been defined for terrestrial

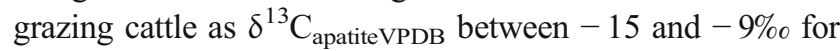
pre-industrial British animals (Gan et al. 2018). The range can be extended to $>-8 \%$ for animals that graze on seaweed (Balasse et al. 2005, 2006). Temperature, altitude, latitude, canopy, and mean annual rainfall (MAR) can all affect the $\delta^{13} \mathrm{C}$ of terrestrial plants (Hare et al. 2018; Kohn 2010). While carbon isotope composition predominantly relates to diet, variation has the potential to shed light on the environments where the cattle were raised.

\section{Materials and methods}

\section{Materials}

The dataset comprises intra-tooth strontium $\left({ }^{87} \mathrm{Sr} /{ }^{86} \mathrm{Sr}\right)$, oxygen $\left(\delta^{18} \mathrm{O}_{\text {carbVSMOW }}\right)$ and carbon $\left(\delta^{13} \mathrm{C}_{\text {apatiteVPDB }}\right)$ values for 55 cattle teeth. The vast majority of teeth $(n=49)$ derive from Durrington Walls, with small numbers of specimens analysed from broadly contemporaneous deposits at West Kennet Palisade Enclosures $(n=4)$, Marden $(n=1)$, and Stonehenge $(n=1)$. The presented data are augmented by 12 from a previous pilot study on Durrington Walls (Viner et al. 2010), to make a total number of 67 specimens.

Durrington Walls is the largest henge monument in Britain, covering around $17 \mathrm{ha}$. It is located on the chalk downlands of southern Britain and is just $3 \mathrm{~km}$ from Stonehenge on the west 
bank of the River Avon (Parker Pearson et al. 2011). Excavations during the Stonehenge Riverside Project found the remains of nine houses, as well as a variety of other features including middens, one of which is very substantial, in addition to the henge itself. An extensive programme of radiocarbon dating has narrowed the main period of settlement activity at the site to around half a century, beginning in 2525$2470 \mathrm{cal} \mathrm{BC}$ and ending in 2480-2440 cal BC (Marshall In prep; Parker Pearson et al. 2011). The main period of settlement at the site overlaps with the erection of the sarsen circle and trilithons at Stonehenge (Parker Pearson et al. 2011). All of the specimens used in this study came from the recent excavations at Durrington Walls, undertaken as part of the Stonehenge Riverside Project between 2004 and 2007. Postexcavation analysis of the animal bone assemblage was carried out at the University of Sheffield, initially as part of the Stonehenge Riverside Project, and then the Feeding Stonehenge Project between 2005 and 2013. The single Stonehenge cattle specimen (SH01) was excavated in 1924 (Cleal et al. 1995, pp. 88, 442, fig. 247). The Marden specimen derives from the excavations described in Wainwright et al (1971), but the site has been subject to new excavations in recent years (Leary et al. 2016). Marden is the second largest henge enclosure in Britain covering an area of 14 ha and is located at the edge of the chalkland c. $14 \mathrm{~km}$ north of Durrington Walls, in the Vale of Pewsey, Wiltshire. West Kennet Palisade Enclosures is a double enclosure site located c. $27 \mathrm{~km}$ north of Durrington Walls and is part of the Avebury complex (Whittle 1997; Bayliss et al. 2017). All sites are founded on chalk lithology.

All the analysed specimens derive from secure contexts and can be confidently defined as of Late Neolithic date, the majority deriving from the rich midden deposits at Durrington Walls (Viner et al. 2010). For oxygen and carbon isotope analysis, the number of incremental samples varied from five to 13 depending on dental attrition. Two samples were analysed from each of the newly selected cattle for strontium. Details of the sampling methods are provided below. In addition, a single human tooth (DUR50) from Durrington Walls was analysed. This tooth (an upper second premolar) derived from the surface of a ceremonial avenue (context 585) leading from the timber Southern Circle to the River Avon.

\section{Analytical methods}

Cattle mandibular third $\left(\mathrm{M}_{3}\right)$ molars were selected for analysis. The lingual cusp of the anterior pillar of each tooth was removed, abraded to remove calculus and debris, and sliced transversally at approximately 3-mm intervals (Appendix Figure 1 in the ESM). Between five and 13 enamel slices were obtained per tooth depending on the degree of dental wear. The sequential numbering of the slices was from root-enamel junction to the occlusal surface. The slice numbers are given in the tables. Two slices, one from close to REJ and one from the centre of slice sequence, were used for $\mathrm{Sr}$ isotope analysis. Oxygen isotope analysis was undertaken on alternate slices taken down length of the tooth. Up to six slices per tooth were analysed to represent the range of oxygen compositions within the tooth. Enamel was separated from dentine mechanically at the University of Sheffield and then transferred to the NERC Isotope Geosciences Laboratory at Keyworth to complete the process. The single human sample was removed from the root-enamel junction on the buccal side of the tooth and treated in the same way as the cattle enamel samples.

For $\mathrm{Sr}$ isotope analysis, the enamel samples were cleaned ultrasonically in high purity water, then rinsed twice in high purity water and high purity acetone. They were then weighed into pre-cleaned Teflon beakers and mixed with ${ }^{84} \mathrm{Sr}$ tracer solution and dissolved in Teflon distilled nitric acid $(8 \mathrm{M}$ $\mathrm{HNO}_{3}$ ). Strontium was collected using Eichrom AG50 X8 resin columns and then loaded onto single rhenium filaments following the method of Birck (1986). The isotope composition and concentrations were determined by thermal ionisation mass spectrometry (TIMS). The international standard NBS 987 for ${ }^{87} \mathrm{Sr} /{ }^{86} \mathrm{Sr}$ gave a value of $0.710253 \pm 0.00006$ for static analysis ( $1 \mathrm{SD}, n=350$ ).

For the isotopic analysis of carbonate oxygen, the 10-mg enamel sample was reduced to a fine powder using an agate mortar and pestle. Approximately $3 \mathrm{mg}$ of the enamel powder was loaded into a glass vial and sealed with septa. The vials were transferred to a hot block at $90{ }^{\circ} \mathrm{C}$ on the GV Multiprep system. The vials were evacuated and four drops of anhydrous phosphoric acid were added. The resultant $\mathrm{CO}_{2}$ was collected cryogenically for $14 \mathrm{~min}$ and transferred to a GV IsoPrime dual inlet mass spectrometer. The isotope values are treated as a carbonate. $\delta^{18} \mathrm{O}$ is reported as per mil $(\% \circ)\left({ }^{18} \mathrm{O} /{ }^{16} \mathrm{O}\right)$ normalised to the PDB scale using a within-run calcite laboratory standard (KCM) calibrated against SRM19 and NIST reference material and were converted to the VSMOW scale using the published conversion equation of Coplen (1988): VSMOW $=\left(1.03091 \times \delta^{18} \mathrm{O}_{\mathrm{VPDB}}\right)+30.91$. Analytical reproducibility for laboratory standard calcite $(\mathrm{KCM})$ is for $\delta^{18} \mathrm{O}_{\text {VSMOW }}= \pm 0.05 \%$ o $(1 \sigma, n=20)$ and $\delta^{13} \mathrm{C}_{\mathrm{VPDB}}$ is \pm $0.03 \% o(1 \sigma, n=20)$ and analytical reproducibility for an inhouse tooth enamel standard is $\delta^{18} \mathrm{O}_{\mathrm{VSMOW}}= \pm 0.32 \%$ o $(1 \sigma$, $n=9)$ and $\delta^{13} \mathrm{C}_{\mathrm{VPDB}}$ is $\pm 0.17 \%$ o $(1 \sigma, n=9)$.

\section{Results and discussion}

\section{Oxygen and carbon isotope data}

The stable $\delta^{18} \mathrm{O}_{\text {carbVSMOW }}$ and $\delta^{13} \mathrm{C}_{\text {carbVPDB }}$ isotope data for the incremental enamel samples are given in Table 1 and summary statistics are given in Table 2 and displayed using box and whisker charts in Figs. 2 and 3. 
Table 1 The $\delta^{13} \mathrm{C}_{\text {carbVPDB }}$ and $\delta^{18} \mathrm{O}_{\text {carbvSMow }}$ composition of tooth slices from Durrington Walls, Marden, West Kennet Palisade Enclosures, and Stonehenge

\begin{tabular}{|c|c|c|c|}
\hline Sample & Slice & $\delta^{13} \mathrm{C}_{\text {carbVPDB }}$ & $\delta^{18} \mathrm{O}_{\text {carbvSMOW }}$ \\
\hline DUR 01 & 3 & -13.72 & 25.41 \\
\hline DUR 01 & 5 & -13.65 & 25.70 \\
\hline DUR 01 & 7 & -13.88 & 24.12 \\
\hline DUR 01 & 9 & -13.26 & 24.25 \\
\hline DUR 01 & 10 & -13.27 & 25.89 \\
\hline DUR 02 & 3 & -13.31 & 26.31 \\
\hline DUR 02 & 5 & -13.38 & 26.35 \\
\hline DUR 02 & 7 & -13.45 & 25.32 \\
\hline DUR 02 & 9 & -13.39 & 24.91 \\
\hline DUR 02 & 10 & -13.15 & 25.29 \\
\hline DUR 03 & 1 & -14.57 & 25.23 \\
\hline DUR 03 & 3 & -14.67 & 26.00 \\
\hline DUR 03 & 5 & -14.25 & 25.56 \\
\hline DUR 03 & 7 & -14.01 & 24.65 \\
\hline DUR 04 & 3 & -13.75 & 26.63 \\
\hline DUR 04 & 5 & -13.99 & 25.49 \\
\hline DUR 04 & 7 & -14.07 & 24.62 \\
\hline DUR 04 & 9 & -13.55 & 24.91 \\
\hline DUR 05 & 1 & -13.79 & 25.36 \\
\hline DUR 05 & 3 & -13.99 & 24.67 \\
\hline DUR 05 & 5 & -14.14 & 24.38 \\
\hline DUR 05 & 7 & -14.11 & 24.95 \\
\hline DUR 06 & 5 & -14.02 & 25.82 \\
\hline DUR 06 & 7 & -14.13 & 25.24 \\
\hline DUR 06 & 9 & -13.84 & 25.48 \\
\hline DUR 07 & 3 & -13.80 & 24.78 \\
\hline DUR 07 & 5 & -14.50 & 26.03 \\
\hline DUR 07 & 7 & -14.88 & 23.27 \\
\hline DUR 07 & 9 & -14.61 & 26.81 \\
\hline DUR 08 & 3 & -14.08 & 25.77 \\
\hline DUR 08 & 5 & -14.22 & 26.00 \\
\hline DUR 08 & 7 & -15.95 & 25.70 \\
\hline DUR 08 & 9 & -14.65 & 27.29 \\
\hline DUR 09 & 1 & -13.90 & 24.80 \\
\hline DUR 09 & 3 & -13.83 & 25.75 \\
\hline DUR 09 & 5 & -14.03 & 24.72 \\
\hline DUR 09 & 7 & -14.06 & 24.43 \\
\hline DUR 09 & 9 & -14.00 & 24.56 \\
\hline DUR 09 & 11 & -14.11 & 25.74 \\
\hline DUR 10 & 1 & -13.62 & 24.59 \\
\hline DUR 10 & 3 & -13.66 & 23.11 \\
\hline DUR 10 & 5 & -13.32 & 23.74 \\
\hline DUR 11 & 1 & -13.80 & 25.77 \\
\hline DUR 11 & 3 & -13.94 & 25.02 \\
\hline DUR 11 & 5 & -13.45 & 24.23 \\
\hline DUR 12 & 1 & -13.55 & 25.41 \\
\hline DUR 12 & 3 & -13.52 & 25.24 \\
\hline
\end{tabular}

Table 1 (continued)

\begin{tabular}{|c|c|c|c|}
\hline Sample & Slice & $\delta^{13} \mathrm{C}_{\text {carbVPDB }}$ & $\delta^{18} \mathrm{O}_{\text {carbVSMOw }}$ \\
\hline DUR 12 & 5 & -13.39 & 23.86 \\
\hline DUR 12 & 7 & -13.56 & 24.55 \\
\hline DUR 12 & 9 & -13.09 & 24.84 \\
\hline DUR 13 & 1 & -13.09 & 24.94 \\
\hline DUR 13 & 3 & -13.63 & 26.07 \\
\hline DUR 13 & 5 & -13.68 & 25.90 \\
\hline DUR 13 & 7 & -13.70 & 25.29 \\
\hline DUR 14 & 1 & -13.31 & 25.46 \\
\hline DUR 14 & 3 & -13.79 & 24.56 \\
\hline DUR 14 & 5 & -13.87 & 23.09 \\
\hline DUR 14 & 7 & -13.48 & 24.67 \\
\hline DUR 15 & 1 & -13.44 & 26.04 \\
\hline DUR 15 & 3 & -13.58 & 25.43 \\
\hline DUR 15 & 5 & -13.74 & 24.99 \\
\hline DUR 16 & 1 & -13.38 & 25.76 \\
\hline DUR 16 & 3 & -13.53 & 25.43 \\
\hline DUR 16 & 5 & -13.47 & 24.09 \\
\hline DUR 17 & 1 & -14.57 & 25.12 \\
\hline DUR 17 & 3 & -14.19 & 25.31 \\
\hline DUR 17 & 5 & -13.38 & 24.90 \\
\hline DUR 18 & 1 & -13.52 & 26.13 \\
\hline DUR 18 & 3 & -13.50 & 25.65 \\
\hline DUR 18 & 5 & -13.54 & 25.00 \\
\hline DUR 19 & 1 & -13.85 & 25.30 \\
\hline DUR 19 & 3 & -13.80 & 24.88 \\
\hline DUR 19 & 5 & -13.89 & 23.88 \\
\hline DUR 19 & 7 & -14.03 & 23.79 \\
\hline DUR 19 & 9 & -13.86 & 24.30 \\
\hline DUR21 & 1 & -13.68 & 23.82 \\
\hline DUR21 & 3 & -13.59 & 24.34 \\
\hline DUR21 & 5 & -13.62 & 24.35 \\
\hline DUR21 & 7 & -13.45 & 23.46 \\
\hline DUR 22 & 1 & -13.78 & 24.10 \\
\hline DUR 22 & 3 & -14.15 & 24.36 \\
\hline DUR 22 & 5 & -14.27 & 24.94 \\
\hline DUR 23 & 6 & -14.12 & 26.23 \\
\hline DUR 23 & 8 & -14.40 & 25.99 \\
\hline DUR 23 & 10 & -14.34 & 25.03 \\
\hline DUR 23 & 12 & -14.39 & 25.30 \\
\hline DUR 24 & 1 & -14.66 & 24.85 \\
\hline DUR 24 & 3 & -14.72 & 25.81 \\
\hline DUR 24 & 5 & -14.80 & 25.96 \\
\hline DUR 24 & 7 & -14.92 & 25.66 \\
\hline DUR 24 & 9 & -15.08 & 24.78 \\
\hline DUR 25 & 1 & -14.03 & 24.86 \\
\hline DUR 25 & 3 & -14.26 & 25.53 \\
\hline DUR 25 & 5 & -14.18 & 25.65 \\
\hline DUR 25 & 7 & -14.21 & 25.91 \\
\hline DUR 26 & 1 & -13.20 & 26.80 \\
\hline
\end{tabular}


Table 1 (continued)

\begin{tabular}{|c|c|c|c|}
\hline Sample & Slice & $\delta^{13} \mathrm{C}_{\text {carbVPDB }}$ & $\delta^{18} \mathrm{O}_{\text {carbVSMOW }}$ \\
\hline DUR 26 & 3 & -13.21 & 26.64 \\
\hline DUR 26 & 5 & -13.79 & 26.69 \\
\hline DUR 26 & 7 & -14.08 & 26.25 \\
\hline DUR 26 & 9 & -14.25 & 26.14 \\
\hline DUR 27 & 1 & -13.68 & 25.20 \\
\hline DUR 27 & 3 & -13.68 & 25.47 \\
\hline DUR 27 & 5 & -13.74 & 25.24 \\
\hline DUR 27 & 7 & -13.87 & 24.44 \\
\hline DUR 28 & 6 & -14.60 & 25.00 \\
\hline DUR 28 & 8 & -14.79 & 24.89 \\
\hline DUR 28 & 10 & -14.73 & 25.05 \\
\hline DUR 28 & 12 & -15.11 & 25.81 \\
\hline DUR 29 & 1 & -13.46 & 26.46 \\
\hline DUR 29 & 3 & -13.31 & 26.11 \\
\hline DUR 29 & 5 & -13.79 & 27.19 \\
\hline DUR 29 & 9 & -13.50 & 26.45 \\
\hline DUR 29 & 11 & -13.27 & 26.12 \\
\hline DUR 30 & 1 & -13.85 & 25.95 \\
\hline DUR 30 & 3 & -14.16 & 25.85 \\
\hline DUR 30 & 5 & -13.97 & 26.68 \\
\hline DUR 30 & 7 & -13.62 & 25.13 \\
\hline DUR 30 & 9 & -13.64 & 24.84 \\
\hline DUR 30 & 11 & -13.45 & 24.76 \\
\hline DUR 31 & 3 & -13.60 & 26.77 \\
\hline DUR 31 & 5 & -13.74 & 26.53 \\
\hline DUR 31 & 7 & -13.73 & 26.76 \\
\hline DUR 31 & 9 & -14.05 & 26.97 \\
\hline DUR 32 & 1 & -14.41 & 25.61 \\
\hline DUR 32 & 3 & -14.57 & 24.59 \\
\hline DUR 32 & 5 & -14.76 & 24.23 \\
\hline DUR 33 & 1 & -13.72 & 25.39 \\
\hline DUR 33 & 3 & -13.95 & 25.14 \\
\hline DUR 33 & 5 & -14.30 & 25.84 \\
\hline DUR 33 & 7 & -14.74 & 26.11 \\
\hline DUR 33 & 9 & -14.25 & 26.38 \\
\hline DUR 34 & 5 & -14.12 & 25.55 \\
\hline DUR 34 & 7 & -14.37 & 24.95 \\
\hline DUR 35 & 1 & -12.40 & 25.21 \\
\hline DUR 35 & 3 & -12.58 & 24.89 \\
\hline DUR 35 & 5 & -13.23 & 24.09 \\
\hline DUR 36 & 1 & -11.83 & 24.11 \\
\hline DUR 36 & 3 & -12.29 & 24.71 \\
\hline DUR 36 & 4 & -12.24 & 24.81 \\
\hline DUR 37 & 1 & -13.67 & 25.50 \\
\hline DUR 37 & 3 & -13.56 & 24.93 \\
\hline DUR 37 & 5 & -13.50 & 24.82 \\
\hline DUR 37 & 7 & -14.00 & 24.12 \\
\hline DUR 37 & 9 & -13.95 & 24.44 \\
\hline DUR 38 & 1 & -12.61 & 26.00 \\
\hline
\end{tabular}

Table 1 (continued)

\begin{tabular}{|c|c|c|c|}
\hline Sample & Slice & $\delta^{13} \mathrm{C}_{\text {carbVPDB }}$ & $\delta^{18} \mathrm{O}_{\text {carbVSMOw }}$ \\
\hline DUR 38 & 3 & -12.60 & 25.70 \\
\hline DUR 38 & 5 & -12.89 & 25.02 \\
\hline DUR 38 & 7 & -13.07 & 24.90 \\
\hline DUR 38 & 9 & -13.44 & 24.69 \\
\hline DUR 39 & 7 & -13.82 & 23.93 \\
\hline DUR 39 & 9 & -13.46 & 24.45 \\
\hline DUR 39 & 11 & -13.96 & 25.33 \\
\hline DUR 39 & 13 & -14.21 & 25.51 \\
\hline DUR 40 & 1 & -13.93 & 26.01 \\
\hline DUR 40 & 3 & -14.05 & 26.05 \\
\hline DUR 40 & 5 & -14.70 & 25.32 \\
\hline DUR 40 & 7 & -15.13 & 23.97 \\
\hline DUR 41 & 1 & -13.07 & 26.20 \\
\hline DUR 41 & 5 & -13.07 & 24.83 \\
\hline DUR 41 & 7 & -12.94 & 24.64 \\
\hline DUR 42 & 1 & -14.20 & 24.27 \\
\hline DUR 42 & 3 & -14.15 & 24.68 \\
\hline DUR 42 & 5 & -13.97 & 23.33 \\
\hline DUR 43 & 1 & -13.57 & 26.34 \\
\hline DUR 43 & 3 & -14.26 & 25.72 \\
\hline DUR 43 & 5 & -14.09 & 24.87 \\
\hline DUR 43 & 7 & -14.46 & 24.20 \\
\hline DUR 43 & 9 & -13.99 & 25.61 \\
\hline DUR 44 & 1 & -14.42 & 26.76 \\
\hline DUR 44 & 3 & -14.30 & 27.38 \\
\hline DUR 44 & 5 & -13.89 & 26.19 \\
\hline DUR 44 & 7 & -13.89 & 25.04 \\
\hline DUR 44 & 9 & -13.85 & 25.16 \\
\hline DUR 45 & 1 & -12.14 & 25.13 \\
\hline DUR 45 & 3 & -11.89 & 24.94 \\
\hline DUR 45 & 5 & -11.62 & 25.94 \\
\hline DUR 45 & 7 & -11.84 & 26.03 \\
\hline DUR 45 & 9 & -12.62 & 24.65 \\
\hline DUR 46 & 1 & -13.09 & 24.65 \\
\hline DUR 46 & 3 & -13.29 & 25.02 \\
\hline DUR 46 & 5 & -13.25 & 25.23 \\
\hline DUR 46 & 7 & -12.83 & 24.06 \\
\hline DUR 46 & 9 & -12.97 & 23.90 \\
\hline DUR 47 & 1 & -13.00 & 25.99 \\
\hline DUR 47 & 3 & -13.11 & 25.61 \\
\hline DUR 47 & 5 & -13.42 & 25.42 \\
\hline DUR 47 & 7 & -13.19 & 24.87 \\
\hline DUR 47 & 9 & -13.58 & 26.01 \\
\hline DUR 47 & 11 & -13.90 & 26.15 \\
\hline DUR 48 & 1 & -13.20 & 24.41 \\
\hline DUR 48 & 3 & -13.71 & 25.48 \\
\hline DUR 48 & 5 & -13.90 & 24.90 \\
\hline DUR 48 & 7 & -13.84 & 25.28 \\
\hline DUR 49 & 1 & -13.03 & 23.73 \\
\hline
\end{tabular}


Table 1 (continued)

\begin{tabular}{|c|c|c|c|}
\hline Sample & Slice & $\delta^{13} \mathrm{C}_{\text {carbVPDB }}$ & $\delta^{18} \mathrm{O}_{\text {carbVSMow }}$ \\
\hline DUR 49 & 3 & -13.15 & 24.66 \\
\hline DUR 49 & 5 & -13.65 & 25.23 \\
\hline DUR 49 & 7 & -14.03 & 25.41 \\
\hline DUR 49 & 9 & -14.15 & 25.12 \\
\hline DW 01 & 1 & -13.45 & 25.33 \\
\hline DW 01 & 3 & -13.64 & 25.24 \\
\hline DW 01 & 5 & -13.50 & 24.29 \\
\hline DW 01 & 7 & -13.89 & 24.73 \\
\hline DW 02 & 1 & -14.09 & 25.72 \\
\hline DW 03 & 1 & -13.92 & 25.56 \\
\hline DW 04 & 3 & -14.02 & 25.00 \\
\hline DW 04 & 5 & -13.95 & 24.84 \\
\hline DW 06 & 1 & -14.80 & 25.59 \\
\hline DW 06 & 3 & -14.61 & 25.60 \\
\hline DW 07 & 3 & -13.45 & 24.82 \\
\hline DW 08 & 3 & -13.34 & 25.27 \\
\hline DW 08 & 5 & -13.52 & 25.53 \\
\hline DW 08 & 9 & -13.69 & 24.61 \\
\hline DW 10 & 1 & -13.67 & 25.44 \\
\hline DW 14 & 3 & -13.85 & 26.25 \\
\hline DW 41 & 3 & -12.92 & 25.95 \\
\hline SH 01 & 1 & -12.31 & 25.18 \\
\hline SH 01 & 3 & -12.88 & 25.25 \\
\hline SH 01 & 5 & -13.34 & 24.67 \\
\hline SH 01 & 7 & -14.10 & 24.19 \\
\hline SH 01 & 9 & -13.96 & 23.94 \\
\hline WK 01 & 1 & -13.29 & 25.63 \\
\hline WK 01 & 3 & -13.47 & 25.43 \\
\hline WK 01 & 5 & -13.63 & 24.95 \\
\hline WK 01 & 7 & -13.37 & 24.53 \\
\hline WK 01 & 9 & -13.25 & 24.98 \\
\hline WK 02 & 3 & -13.28 & 27.37 \\
\hline WK 02 & 5 & -13.07 & 26.48 \\
\hline WK 02 & 7 & -13.48 & 25.67 \\
\hline WK 02 & 9 & -13.51 & 23.94 \\
\hline WK 02 & 10 & -13.28 & 26.84 \\
\hline WK 03 & 3 & -11.87 & 26.64 \\
\hline WK 03 & 5 & -11.83 & 26.77 \\
\hline WK 03 & 7 & -12.16 & 26.34 \\
\hline WK 03 & 10 & -11.93 & 25.86 \\
\hline WK 04 & 1 & -13.71 & 25.34 \\
\hline WK 04 & 3 & -13.75 & 24.37 \\
\hline WK 04 & 5 & -13.79 & 24.50 \\
\hline WK 04 & 7 & -13.92 & 24.87 \\
\hline WK 04 & 9 & -14.02 & 25.28 \\
\hline
\end{tabular}

The median $\delta^{18} \mathrm{O}_{\text {carbvsmow }}$ intra-tooth values range between $23.74 \%$ (DUR 10) and $26.77 \%$ (DUR 31). The interquartile range (IQR: the central $50 \%$ of the data range) of the $\delta^{18} \mathrm{O}_{\text {carbvsmow values ranges widely. Of the animals }}$ that have three or more slices measured per tooth, DUR 31 has the smallest IQR of 0.33 and DUR 7 has the largest IQR of 2.97. The typical IQR seasonal range in British cattle, as represented by the Chillingham herd, is 1.23 . This value can be taken to represent the natural variation in drinking water in a static herd. This suggests that animals with a substantially lower IQR (i.e. less variation) probably relied on a more seasonally stable water source, such as aquifer-sourced rivers or deep, low evaporation lakes. Alternatively, they could have been subject to seasonal movement that would dampen the impact of intra-annual variation in water oxygen (e.g. summer upland and winter lowland pasture). Conversely, those animals with a large range of $\delta^{18} \mathrm{O}_{\text {carbvSMOw }}$ in their teeth might be enhancing the range of water oxygen they are exposed to through shallow evaporated ponds and lakes, or may be incorporating a wider range of water oxygen through movement to varied pasturage, as part of either seasonal management regimes or population movement.

The range of carbon isotope values between animals also varies widely. Five samples (DUR 35, DUR 36, DUR 38, DUR 45, and WK3) have $\delta^{13} \mathrm{C}_{\text {carbVPDB }}$ median values above $-13 \%$ o, the highest being $-11.89 \%$ (DUR 45). The lowest median value is $-14.8 \%$ (DUR 24). As in the oxygen data, there are considerable differences in the magnitude of intratooth variation, as reflected in the IQR values. The smallest IQR is 0.04 from three slices (DUR 18) and the highest 1.5 from four slices (DUR 8). The Chillingham animals record an average IQR of 0.4 . These values are within the range -9 and - 15 for pre-industrial terrestrial grazing British cattle (Gan et al. 2018). The differences in interquartile ranges may reflect variation in animal management, those with a small IQR having more constant annual environments, and those with a larger IQR potentially grazing in areas of more variable canopy cover and/or rainfall levels.

The intra-tooth variations in oxygen and carbon reflect a heterogeneity in the manner in which these animals accessed water and plants. This is strongly suggestive of disparate origins with respect to drinking and grazing habits. This withintooth diversity extends, in some degree, to the strontium data described below.

\section{Strontium isotope data}

The Sr isotope variations within the teeth (Tables 2 and 3) are plotted in Fig. 4 in ascending value of the samples from occlusal surfaces (the earliest developing sample), showing the rest of the incremental data relative to this.

Of the 64 cattle teeth that were analysed, only 11 have data that fall at least partially in the range defined for chalk and, of these, only three animals have values that are all consistent with chalk (Table 4). Therefore, results indicate that the 


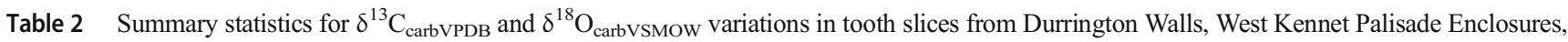
and Stonehenge

\begin{tabular}{|c|c|c|c|c|c|c|c|c|c|c|c|c|c|c|c|c|c|}
\hline $\begin{array}{l}\text { Sample } \\
\text { to Tooth }\end{array}$ & $N^{*}$ & $\begin{array}{l}\text { Mean } \\
\delta^{13} \mathrm{C} \\
\text { carbVPDB }\end{array}$ & StDev & Min & Q1 & Median & Q3 & Max & IQR & $\begin{array}{l}\text { Mean } \\
\delta^{18} \mathrm{O}_{\text {carb }} \\
\text { vSMOW }\end{array}$ & StDev & Min & Q1 & Median & Q3 & Max & IQR \\
\hline DUR 01 & 5 & -13.56 & 0.28 & -13.88 & -13.80 & -13.65 & -13.27 & -13.26 & 0.54 & 25.07 & 0.83 & 24.12 & 24.19 & 25.41 & 25.80 & 25.89 & 1.61 \\
\hline DUR 02 & 5 & -13.34 & 0.12 & -13.45 & -13.42 & -13.38 & -13.23 & -13.15 & 0.19 & 25.64 & 0.65 & 24.91 & 25.10 & 25.32 & 26.33 & 6.35 & 1.23 \\
\hline DUR 03 & 4 & -14.38 & 0.30 & -14.67 & -14.65 & -14.41 & -14.07 & -14.01 & 0.57 & 25.36 & 0.57 & 24.65 & 24.80 & 25.40 & 25.89 & 26.00 & 1.10 \\
\hline OUR 04 & 4 & -13.84 & 0.24 & -14.07 & -14.05 & -13.87 & -13.60 & -13.55 & 0.45 & 25.41 & 0.89 & 24.62 & 24.69 & 25.20 & 26.35 & 26.63 & 1.65 \\
\hline UR 05 & 4 & -14.01 & 0.16 & -14.14 & -14.13 & -14.05 & -13.84 & -13.79 & 0.29 & 24.84 & 0.42 & 24.38 & 24.45 & 24.81 & 25.26 & 25.36 & 0.81 \\
\hline UR 06 & 3 & -14.00 & 0.15 & 14.13 & -14.13 & -14.02 & -13.84 & -13.84 & 0.29 & 25.51 & 0.29 & 25.24 & 25.24 & 25.48 & 25.82 & 25.82 & 0.58 \\
\hline UR 07 & 4 & -14.45 & 0.46 & 14.88 & -14.81 & -14.56 & -13 & -13.80 & 0.84 & 25.22 & 1.55 & 23.27 & 23.65 & 25.41 & 26.62 & 26.81 & 2.97 \\
\hline UR 08 & 4 & -14.73 & 0.85 & 15.95 & -15.63 & -14.44 & -14.12 & -14.08 & 1.51 & 26.19 & 0.74 & 25.70 & 25.72 & 25.89 & 26.97 & 27.29 & 1.25 \\
\hline DUR 09 & 6 & -13.99 & 0.11 & -14.11 & -14.07 & -14.02 & -13.88 & -13.83 & 0.19 & 25.00 & 0.59 & 24.43 & 24.53 & 24.76 & 25.74 & 25.75 & 1.22 \\
\hline DUR 10 & 3 & -13.53 & 0.19 & -13.66 & -13.66 & -13.62 & -13.32 & -13.32 & 0.34 & 23.81 & 0.74 & 23.11 & 23.11 & 23.74 & 24.59 & 24.59 & 1.48 \\
\hline DUR 11 & 3 & -13.73 & 0.25 & -13.94 & -13.94 & -13.80 & -13.45 & -13.45 & 0.49 & 25.01 & 0.77 & 24.23 & 24.23 & 25.02 & 25.77 & 25.77 & 1.54 \\
\hline DUR 12 & 5 & -13.42 & 0.20 & -13.56 & -13.56 & -13.52 & -13.24 & -13.09 & 0.32 & 24.78 & 0.62 & 23.86 & 24.21 & 24.84 & 25.33 & 25.41 & 1.12 \\
\hline DUR 13 & 4 & -13.53 & 0.29 & -13.70 & -13.70 & -13.66 & -13.23 & -13.09 & 0.47 & 25.55 & 0.53 & 24.94 & 25.03 & 25.60 & 26.03 & 26.07 & 1.00 \\
\hline OUR 14 & 4 & -13.61 & 0.26 & -13.87 & -13.85 & -13.64 & -13.35 & -13.31 & 0.50 & 24.45 & 0.99 & 23.09 & 23.46 & 24.62 & 25.26 & 25.46 & 1.80 \\
\hline OUR 15 & 3 & -13.59 & 0.15 & -13.74 & -13.74 & -13.58 & -13.44 & -13.44 & 0.30 & 25.49 & 0.53 & 24.99 & 24.99 & 25.43 & 26.04 & 26.04 & 1.05 \\
\hline UR 16 & 3 & -13.46 & 0.08 & -13.53 & -13.53 & -13.47 & -13.38 & -13.38 & 0.15 & 25.09 & 0.88 & 24.09 & 24.09 & 25.43 & 25.76 & 25.76 & 1.67 \\
\hline UR 17 & 3 & -14.05 & 0.61 & -14.57 & -14.57 & -14.19 & -13.38 & -13.38 & 1.19 & 25.11 & 0.21 & 24.90 & 24.90 & 25.12 & 25.31 & 25.31 & 0.41 \\
\hline UR 18 & 3 & -13.52 & 0.02 & -13.54 & -13.54 & -13.52 & -13.50 & -13.50 & 0.04 & 25.59 & 0.57 & 25.00 & 25.00 & 25.65 & 26.13 & 26.13 & 1.13 \\
\hline UR 19 & 5 & -13.89 & 0.09 & -14.03 & -13.96 & -13.86 & -13.83 & -13.80 & 0.14 & 24.43 & 0.65 & 23.79 & 23.84 & 24.30 & 25.09 & 25.30 & 1.26 \\
\hline OUR 21 & 4 & -13.59 & 0.10 & -13.68 & -13.67 & -13.61 & -13.49 & -13.45 & 0.18 & 23.99 & 0.43 & 23.46 & 23.55 & 24.08 & 24.35 & 24.35 & 0.80 \\
\hline DUR 22 & 3 & -14.07 & 0.26 & -14.27 & -14.27 & -14.15 & -13.78 & -13.78 & 0.49 & 24.47 & 0.43 & 24.10 & 24.10 & 24.36 & 24.94 & 24.94 & 0.84 \\
\hline UUR 23 & 4 & -14.31 & 0.13 & -14.40 & -14.40 & -14.37 & -14.18 & -14.12 & 0.22 & 25.64 & 0.57 & 25.03 & 25.10 & 25.65 & 26.17 & 26.23 & 1.07 \\
\hline OUR 24 & 5 & -14.84 & 0.17 & -15.08 & -15.00 & -14.80 & -14.69 & -14.66 & 0.31 & 25.41 & 0.56 & 24.78 & 24.82 & 25.66 & 25.89 & 25.96 & 1.07 \\
\hline UUR 25 & 4 & -14.17 & 0.10 & -14.26 & -14.25 & -14.20 & -14.07 & -14.03 & 0.18 & 25.49 & 0.45 & 24.86 & 25.03 & 25.59 & 25.85 & 25.91 & 0.82 \\
\hline UR 26 & 5 & -13.71 & 0.49 & -14.25 & -14.17 & -13.79 & -13.21 & -13.20 & 0.96 & 26.50 & 0.29 & 26.14 & 26.20 & 26.64 & 26.75 & 26.80 & 0.55 \\
\hline UR 27 & 4 & -13.74 & 0.09 & -13.87 & -13.84 & -13.71 & -13.68 & -13.68 & 0.16 & 25.09 & 0.45 & 24.44 & 24.63 & 25.22 & 25.41 & 25.47 & 0.78 \\
\hline UUR 28 & 4 & -14.81 & 0.22 & -15.11 & -15.03 & -14.76 & -14.63 & -14.60 & 0.40 & 25.19 & 0.42 & 24.89 & 24.92 & 25.03 & 25.62 & 25.81 & 0.70 \\
\hline DUR 29 & 5 & -13.47 & 0.21 & -13.79 & -13.65 & -13.46 & -13.29 & -13.27 & 0.36 & 26.47 & 0.44 & 26.11 & 26.12 & 26.45 & 26.83 & 27.19 & 0.71 \\
\hline DUR 30 & 6 & -13.78 & 0.26 & -14.16 & -14.02 & -13.75 & -13.58 & -13.45 & 0.44 & 25.54 & 0.75 & 24.76 & 24.82 & 25.49 & 26.13 & 26.68 & 1.31 \\
\hline DUR 31 & 4 & -13.78 & 0.19 & -14.05 & -13.97 & -13.74 & -13.63 & -13.60 & 0.34 & 26.76 & 0.18 & 26.53 & 26.59 & 26.77 & 26.92 & 26.97 & 0.33 \\
\hline DUR 32 & 3 & -14.58 & 0.18 & -14.76 & -14.76 & -14.57 & -14.41 & -14.41 & 0.35 & 24.81 & 0.72 & 24.23 & 24.23 & 24.59 & 25.61 & 25.61 & 1.38 \\
\hline DUR 33 & 5 & -14.19 & 0.39 & -14.74 & -14.52 & -14.25 & -13.84 & -13.72 & 0.68 & 25.77 & 0.51 & 25.14 & 25.27 & 25.84 & 26.25 & 26.38 & 0.98 \\
\hline DUR 34 & 2 & -14.25 & 0.18 & -14.37 & & -14.25 & & -14.12 & & 25.25 & 0.42 & 24.95 & & 25.25 & & 25.55 & \\
\hline DUR 35 & 3 & -12.74 & 0.44 & -13.23 & -13.23 & -12.58 & -12.40 & -12.40 & 0.83 & 24.73 & 0.58 & 24.09 & 24.09 & 24.89 & 25.21 & 25.21 & 1.12 \\
\hline DUR 36 & 3 & -12.12 & 0.25 & -12.29 & -12.29 & -12.24 & -11.83 & -11.83 & 0.46 & 24.54 & 0.38 & 24.11 & 24.11 & 24.71 & 24.81 & 24.81 & 0.70 \\
\hline DUR 37 & 5 & -13.74 & 0.23 & -14.00 & -13.98 & -13.67 & -13.53 & -13.50 & 0.45 & 24.76 & 0.52 & 24.12 & 24.28 & 24.82 & 25.22 & 25.50 & 0.93 \\
\hline DUR 38 & 5 & -12.92 & 0.35 & -13.44 & -13.26 & -12.89 & -12.61 & -12.60 & 0.65 & 25.26 & 0.56 & 24.69 & 24.80 & 25.02 & 25.85 & 26.00 & 1.06 \\
\hline DUR 39 & 4 & -13.86 & 0.31 & -14.21 & -14.15 & -13.89 & -13.55 & -13.46 & 0.60 & 24.81 & 0.75 & 23.93 & 24.06 & 24.89 & 25.47 & 25.51 & 1.41 \\
\hline DUR 40 & 4 & -14.45 & 0.56 & -15.13 & -15.02 & -14.38 & -13.96 & -13.93 & 1.06 & 25.34 & 0.97 & 23.97 & 24.31 & 25.67 & 26.04 & 26.05 & 1.73 \\
\hline DUR 41 & 3 & -13.03 & 0.08 & -13.07 & -13.07 & -13.07 & -12.94 & -12.94 & 0.13 & 25.22 & 0.85 & 24.64 & 24.64 & 24.83 & 26.20 & 26.20 & 1.56 \\
\hline DUR 42 & 3 & -14.11 & 0.12 & -14.20 & -14.20 & -14.15 & -13.97 & -13.97 & 0.23 & 24.09 & 0.69 & 23.33 & 23.33 & 24.27 & 24.68 & 24.68 & 1.35 \\
\hline DUR 43 & 5 & -14.07 & 0.33 & -14.46 & -14.36 & -14.09 & -13.78 & -13.57 & 0.58 & 25.35 & 0.83 & 24.20 & 24.54 & 25.61 & 26.03 & 26.34 & 1.50 \\
\hline DUR 44 & 5 & -14.07 & 0.27 & -14.42 & -14.36 & -13.89 & -13.87 & -13.85 & 0.49 & 26.11 & 1.01 & 25.04 & 25.10 & 26.19 & 27.07 & 27.38 & 1.97 \\
\hline DUR 45 & 5 & -12.02 & 0.38 & -12.62 & -12.38 & -11.89 & -11.73 & -11.62 & 0.65 & 25.34 & 0.62 & 24.65 & 24.80 & 25.13 & 25.99 & 26.03 & 1.19 \\
\hline DUR 46 & 5 & -13.09 & 0.19 & -13.29 & -13.27 & -13.09 & -12.90 & -12.83 & 0.37 & 24.57 & 0.58 & 23.90 & 23.98 & 24.65 & 25.13 & 25.23 & 1.15 \\
\hline DUR 47 & 6 & -13.37 & 0.34 & -13.90 & -13.66 & -13.31 & -13.08 & -13.00 & 0.58 & 25.68 & 0.48 & 24.87 & 25.28 & 25.80 & 26.05 & 26.15 & 0.76 \\
\hline
\end{tabular}


Table 2 (continued)

\begin{tabular}{|c|c|c|c|c|c|c|c|c|c|c|c|c|c|c|c|c|c|}
\hline $\begin{array}{l}\text { Sample } \\
\text { to Tooth }\end{array}$ & $N^{*}$ & $\begin{array}{l}\text { Mean } \\
\delta^{13} \mathrm{C} \\
\text { carbVPDB }\end{array}$ & StDev & Min & Q1 & Median & Q3 & Max & IQR & $\begin{array}{l}\text { Mean } \\
\delta^{18} \mathrm{O}_{\text {carb }} \\
\text { vsMow }\end{array}$ & StDev & Min & Q1 & Median & Q3 & Max & IQR \\
\hline DUR 48 & 4 & -13.66 & 0.32 & -13.90 & -13.89 & -13.78 & -13.33 & -13.20 & 0.56 & 25.02 & 0.47 & 24.41 & 24.53 & 25.09 & 25.43 & 25.48 & 0.90 \\
\hline DUR 49 & 5 & -13.60 & 0.50 & -14.15 & -14.09 & -13.65 & -13.09 & -13.03 & 1.00 & 24.83 & 0.67 & 23.73 & 24.20 & 25.12 & 25.32 & 25.41 & 1.13 \\
\hline DW 01 & 4 & -13.62 & 0.20 & -13.89 & -13.83 & -13.57 & -13.46 & -13.45 & 0.37 & 24.90 & 0.48 & 24.29 & 24.40 & 24.99 & 25.31 & 25.33 & 0.91 \\
\hline DW 02 & 1 & -14.09 & & -14.09 & & -14.09 & & -14.09 & & 25.72 & & 25.72 & & 25.72 & & 25.72 & \\
\hline DW 03 & 1 & -13.92 & & -13.92 & & -13.92 & & -13.92 & & 25.56 & & 25.56 & & 25.56 & & 25.56 & \\
\hline DW 04 & 2 & -13.99 & 0.05 & -14.02 & & -13.99 & & -13.95 & & 24.92 & 0.11 & 24.84 & & 24.92 & & 25.00 & \\
\hline DW 06 & 2 & -14.71 & 0.13 & -14.80 & & -14.71 & & -14.61 & & 25.60 & 0.01 & 25.59 & & 25.60 & & 25.60 & \\
\hline DW 07 & 1 & -13.45 & & -13.45 & & -13.45 & & -13.45 & & 24.82 & & 24.82 & & 24.82 & & 24.82 & \\
\hline DW 08 & 3 & -13.52 & 0.18 & -13.69 & -13.69 & -13.52 & -13.34 & -13.34 & 0.35 & 25.14 & 0.47 & 24.61 & 24.61 & 25.27 & 25.53 & 25.53 & 0.92 \\
\hline DW 10 & 1 & -13.67 & & -13.67 & & -13.67 & & -13.67 & & 25.44 & & 25.44 & & 25.44 & & 25.44 & \\
\hline DW 14 & 1 & -13.85 & & -13.85 & & -13.85 & & -13.85 & & 26.25 & & 26.25 & & 26.25 & & 26.25 & \\
\hline DW 41 & 1 & -12.92 & & -12.92 & & -12.92 & & -12.92 & & 25.95 & & 25.95 & & 25.95 & & 25.95 & \\
\hline SH 01 & 5 & -13.32 & 0.75 & -14.10 & -14.03 & -13.34 & -12.60 & -12.31 & 1.44 & 24.65 & 0.58 & 23.94 & 24.07 & 24.67 & 25.22 & 25.25 & 1.15 \\
\hline WK 01 & 5 & -13.40 & 0.15 & -13.63 & -13.55 & -13.37 & -13.27 & -13.25 & 0.28 & 25.10 & 0.43 & 24.53 & 24.74 & 24.98 & 25.53 & 25.63 & 0.79 \\
\hline WK 02 & 5 & -13.32 & 0.18 & -13.51 & -13.50 & -13.28 & -13.18 & -13.07 & 0.32 & 26.06 & 1.34 & 23.94 & 24.81 & 26.48 & 27.11 & 27.37 & 2.30 \\
\hline WK 03 & 4 & -11.95 & 0.15 & -12.16 & -12.10 & -11.90 & -11.84 & -11.83 & 0.26 & 26.40 & 0.40 & 25.86 & 25.98 & 26.49 & 26.74 & 26.77 & 0.76 \\
\hline WK 04 & 5 & -13.84 & 0.13 & -14.02 & -13.97 & -13.79 & -13.73 & -13.71 & 0.24 & 24.87 & 0.44 & 24.37 & 24.44 & 24.87 & 25.31 & 25.34 & 0.88 \\
\hline
\end{tabular}

majority of the animals in this study were not raised on the chalkland surrounding the sites. The vector of change shows no systematic shift towards the chalk values in the later developing part of the teeth. This indicates that the animals' movement to the sites was post-mineralisation and that they were not grazing on chalk terrain for any significant time prior to slaughter. As with the carbon and oxygen results, there are considerable differences between the animals' recorded movements: some animals (e.g. DUR 26, DUR 37, and DUR 47) show no marked difference in isotope composition within their tooth enamel. There is no evidence that these animals grazed on different terrains during the mineralisation of their teeth. Other animals (e.g. DUR 02 and DUR 16) show greater intra-tooth variation and are therefore likely to have grazed on pastures in different geological regions during their developmental years. Overall, most animals show little evidence for having been moved across different regions during early life. Only three cattle of the 42 new individuals, for which multiple $\mathrm{Sr}$ samples were analysed, showed strong evidence for movement. DUR 02, DUR 16, and DUR 20 have intra-tooth differences of more than 0.001 . The only other teeth that exhibit a difference of this magnitude (DUR 03, DUR 32, and DUR 38) have high radiogenic values (> 0.713 ) that may be more variable within a single biosphere. The $\mathrm{Sr}$ data are consistent with the vast majority of individual animals being raised in a single location, though it is clear that the animals derived from a wide-ranging area and were brought to the Stonehenge landscape later in life. This suggests that intra-tooth variation in oxygen is most likely to relate to seasonal variation in water oxygen availability or management strategies, rather than long-distance movement of animals in early life.

In summary, the isotope composition of the cattle provides evidence for wide-ranging origins, but no indication of substantial movement in early life. Some intra-tooth oxygen and carbon values are wide-ranging and others are tightly constrained, indicating that management strategies for the cattle were variable, with some showing far more marked seasonal effect on their biogenic values. The next section will focus on inter-tooth, rather than on intra-tooth, values, in an attempt to explore the origins of the animals.

\section{Establishing the geographic origins of the animals}

Oxygen isotope composition provides a broad-brush method for subdividing the data through comparison with animals of known geographic origin. Figure 5 presents a box and whisker diagram that compares the mean $\delta^{18} \mathrm{O}_{\text {carbvsmow values for }}$ animals from this study with those of animals from eastern and central England (Towers et al. 2010, 2011, 2017). In making this comparison, a number of issues have to be considered, including irregular wear patterns, methodological differences in preparation, possible biases to median value where sample numbers are low, and reliability of place of origin. These issues are discussed and addressed in the appendix in the ESM. 


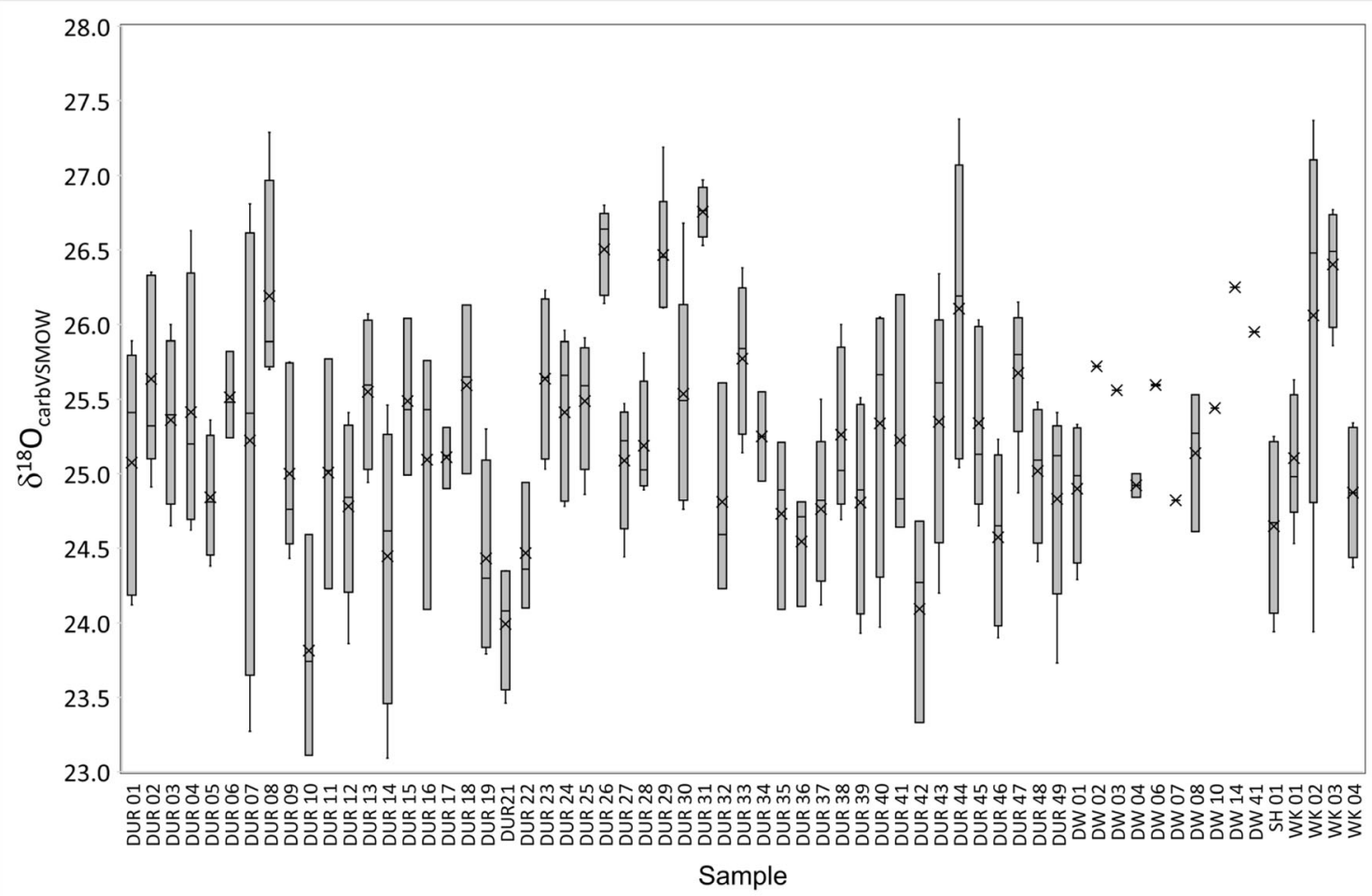

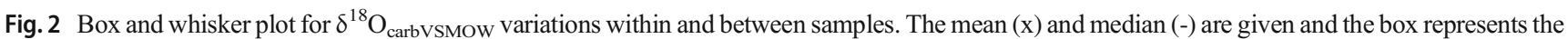
interquartile range (IQR) or central $50 \%$ of the data. The whiskers extend to the maximum and minimum values of the sample dataset

With these caveats in mind, we present the following observations.
There is a clear separation between the data from the two groups, with the animals from this study recording significantly

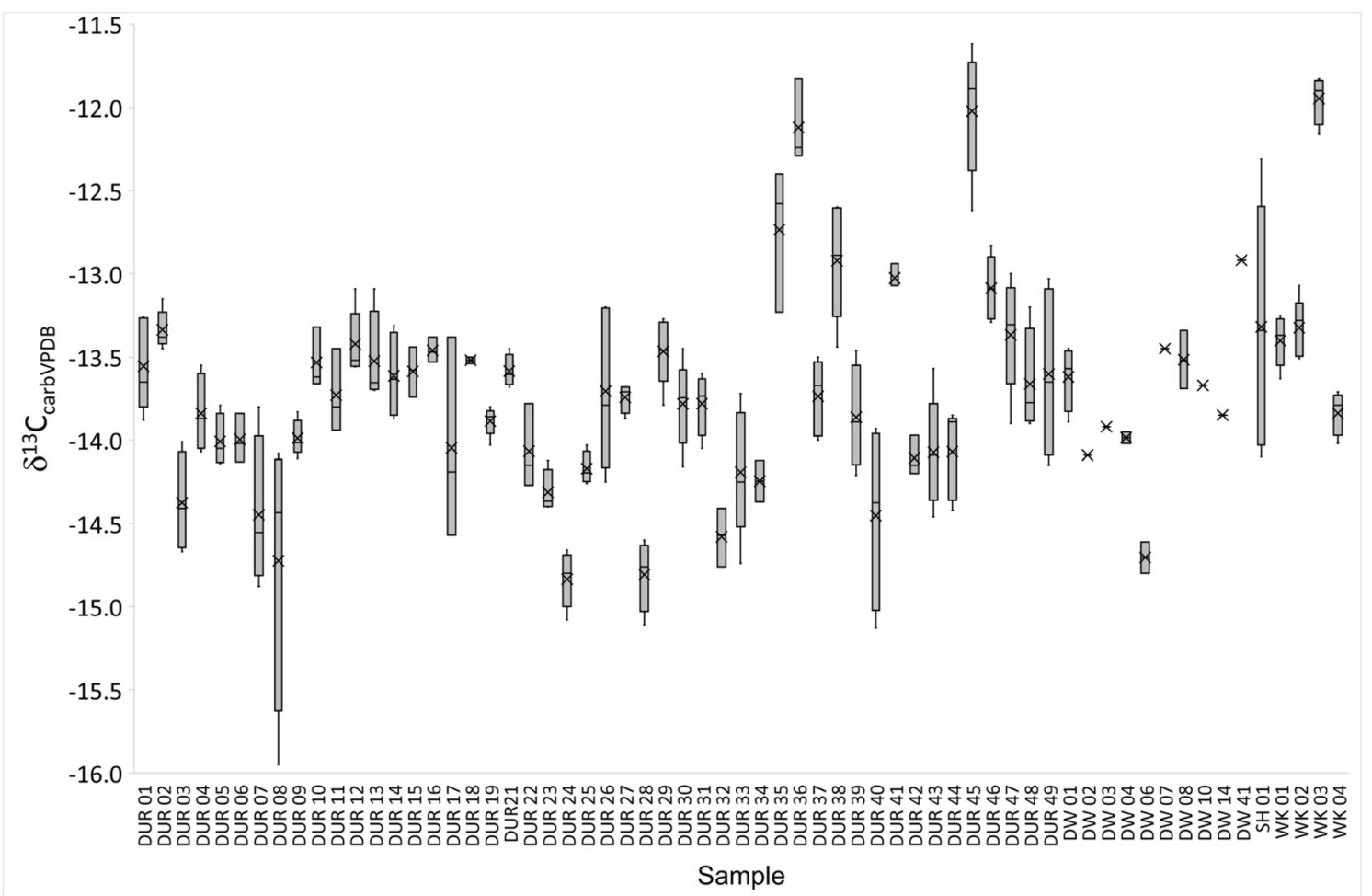

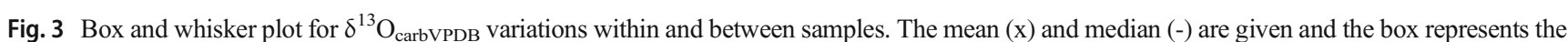
interquartile range (IQR) or central $50 \%$ of the data. The whiskers extent to the maximum and minimum values of the sample dataset 
Table 3 Strontium concentrations and ${ }^{87} \mathrm{Sr} /{ }^{86} \mathrm{Sr}$ values for teeth from Durrington Walls, Marden, West Kennet Palisade Enclosures, and Stonehenge. The table includes previously published DW samples with previously unpublished $\mathrm{Sr}$ concentrations. DUR 50 is a human tooth, while the remainder are cattle teeth

\begin{tabular}{|c|c|c|c|c|c|c|c|}
\hline \multirow{3}{*}{\multicolumn{4}{|c|}{$\begin{array}{l}\text { Stonehenge. The table includes previously published DW samples with } \\
\text { previously unpublished Sr concentrations. DUR } 50 \text { is a human tooth, while } \\
\text { the remainder are cattle teeth }\end{array}$}} & \multirow{4}{*}{$\begin{array}{l}\text { Sample } \\
\text { DUR } 34 \\
\text { DUR } 34\end{array}$} & \multirow{4}{*}{$\begin{array}{l}\text { Slice } \\
5 \\
9\end{array}$} & \multirow{3}{*}{$\begin{array}{l}\text { Sr ppm } \\
126 \\
132\end{array}$} & \multirow{4}{*}{$\begin{array}{l}{ }^{87} \mathrm{Sr} /{ }^{86} \mathrm{Sr} \\
0.70901 \\
0.70932 \\
0.70961\end{array}$} \\
\hline & & & & & & & \\
\hline & & & & & & & \\
\hline Sample & Slice & Sr ppm & ${ }^{87} \mathrm{Sr} /{ }^{86} \mathrm{Sr}$ & & & $\begin{array}{r}132 \\
98\end{array}$ & \\
\hline & & & & DUR 35 & 5 & 107 & 0.70960 \\
\hline SH 1 & 5 & 178 & 0.71264 & DUR 36 & 1 & 66 & 0.71317 \\
\hline MAR 1.1 & 1 & 281 & 0.71034 & DUR 36 & 5 & 64 & 0.71396 \\
\hline WK 01 & 5 & 220 & 0.71128 & DUR 37 & 1 & 124 & 0.71238 \\
\hline WK 02 & 1 & 195 & 0.71139 & DUR 37 & 5 & 129 & 0.71243 \\
\hline WK 02 & 5 & 208 & 0.71068 & DUR 38 & 1 & 139 & 0.71585 \\
\hline WK 03 & 5 & 208 & 0.70948 & DUR 38 & 5 & 112 & 0.71388 \\
\hline WK 04 & 1 & 127 & 0.71020 & DUR 39 & 7 & 138 & 0.70958 \\
\hline WK 04 & 2 & 122 & 0.70974 & DUR 39 & 11 & 133 & 0.70966 \\
\hline DUR 01 & 1 & 126 & 0.70890 & DUR 40 & 1 & 244 & 0.71080 \\
\hline DUR 01 & 5 & 136 & 0.70850 & DUR 40 & 5 & 241 & 0.71090 \\
\hline DUR 02 & 1 & 100 & 0.70845 & DUR 41 & 1 & 157 & 0.70860 \\
\hline DUR 02 & 5 & 122 & 0.70989 & DUR 41 & 5 & 176 & 0.70855 \\
\hline DUR 03 & 1 & 137 & 0.71680 & DUR 42 & 5 & 126 & 0.70976 \\
\hline DUR 03 & 5 & 154 & 0.71498 & DUR 43 & 1 & 184 & 0.70944 \\
\hline DUR 04 & 5 & 136 & 0.71026 & DUR 43 & 5 & 196 & 0.71016 \\
\hline DUR 05 & 5 & 105 & 0.70839 & DUR 44 & 1 & 154 & 0.71035 \\
\hline DUR 06 & 4 & 181 & 0.71003 & DUR 44 & 5 & 144 & 0.70984 \\
\hline DUR 07 & 4 & 186 & 0.71031 & DUR 45 & 1 & 170 & 0.70998 \\
\hline DUR 07 & 7 & 141 & 0.70970 & DUR 45 & 5 & 168 & 0.70976 \\
\hline DUR 09 & 1 & 134 & 0.70920 & DUR 46 & 1 & 159 & 0.71296 \\
\hline DUR 09 & 6 & 106 & 0.70942 & DUR 46 & 5 & 121 & 0.71254 \\
\hline DUR 10 & 1 & 131 & 0.70879 & DUR 47 & 1 & 197 & 0.70893 \\
\hline DUR 10 & 5 & 144 & 0.70832 & DUR 47 & 5 & 250 & 0.70897 \\
\hline DUR 11 & 1 & 114 & 0.70904 & DUR 48 & 1 & 154 & 0.70954 \\
\hline DUR 11 & 5 & 130 & 0.70962 & DUR 48 & 5 & 241 & 0.70971 \\
\hline DUR 12 & 1 & 124 & 0.71234 & DUR 49 & 1 & 119 & 0.70885 \\
\hline DUR 12 & 5 & 128 & 0.71328 & DUR 49 & 5 & 108 & 0.70954 \\
\hline DUR 13 & 1 & 293 & 0.71002 & DUR 50 & & 66 & 0.71261 \\
\hline DUR 13 & 5 & 269 & 0.71011 & DW 01 & 1 & 110 & 0.70976 \\
\hline DUR 14 & 1 & 125 & 0.70921 & DW 01 & 2 & 136 & 0.70966 \\
\hline DUR 14 & 5 & 103 & 0.70964 & DW 01 & 3 & 146 & 0.70959 \\
\hline DUR 15 & 1 & 141 & 0.70946 & DW 02 & 1 & 305 & 0.71260 \\
\hline DUR 15 & 5 & 140 & 0.70956 & DW 02 & 2 & 254 & 0.71255 \\
\hline DUR 16 & 1 & 116 & 0.70916 & DW 02 & 3 & 211 & 0.71139 \\
\hline DUR 16 & 5 & 119 & 0.71057 & DW 03 & 1 & 98 & 0.71025 \\
\hline DUR 17 & 1 & 132 & 0.70865 & DW 03 & 2 & 109 & 0.70955 \\
\hline DUR 17 & 5 & 167 & 0.70905 & DW 03 & 3 & 69 & 0.71084 \\
\hline DUR 18 & 1 & 134 & 0.70948 & DW 04 & 1 & 93 & 0.71359 \\
\hline DUR 18 & 5 & 159 & 0.70922 & DW 04 & 2 & 90 & 0.71308 \\
\hline DUR 19 & 1 & 119 & 0.71376 & DW 04 & 3 & 94 & 0.71207 \\
\hline DUR 19 & 5 & 131 & 0.71347 & DW 05 & 2 & 217 & 0.70923 \\
\hline DUR 20 & 1 & 208 & 0.71068 & DW 05 & 3 & 226 & 0.70893 \\
\hline DUR 20 & 5 & 208 & 0.70948 & DW 06 & 1 & 140 & 0.71017 \\
\hline DUR 21 & 1 & 99 & 0.70933 & DW 06 & 2 & 142 & 0.71043 \\
\hline DUR 22 & 1 & 173 & 0.71062 & DW 07 & 1 & 185 & 0.71069 \\
\hline DUR 22 & 5 & 164 & 0.71034 & DW 07 & 2 & 179 & 0.71062 \\
\hline DUR 23 & 6 & 111 & 0.70811 & DW 07 & 3 & 161 & 0.71055 \\
\hline DUR 24 & 1 & 190 & 0.71008 & DW 08 & 2 & 166 & 0.71052 \\
\hline DUR 24 & 5 & 197 & 0.71048 & DW 08 & 3 & 153 & 0.70981 \\
\hline DUR 26 & 1 & 246 & 0.71005 & DW 08 & 4 & 151 & 0.70950 \\
\hline DUR 26 & 5 & 242 & 0.71011 & DW 09 & 1 & 108 & 0.70872 \\
\hline DUR 28 & 6 & 203 & 0.71070 & DW 09 & 2 & 121 & 0.70881 \\
\hline DUR 28 & 10 & 221 & 0.71056 & DW 09 & 3 & 126 & 0.70893 \\
\hline DUR 29 & 1 & 156 & 0.70890 & DW 10 & 1 & 183 & 0.71050 \\
\hline DUR 29 & 5 & 164 & 0.70899 & DW 10 & 2 & 126 & 0.71133 \\
\hline DUR 30 & 1 & 182 & 0.70929 & DW 10 & 3 & 96 & 0.71220 \\
\hline DUR 30 & 5 & 161 & 0.70995 & DW 11 & 1 & 257 & 0.71094 \\
\hline DUR 31 & 1 & 208 & 0.71082 & DW 11 & 2 & 219 & 0.71066 \\
\hline DUR 31 & 5 & 189 & 0.70999 & DW 11 & 3 & 210 & 0.71082 \\
\hline DUR 32 & 1 & 133 & 0.71329 & DW 12 & 1 & 218 & 0.71509 \\
\hline DUR 32 & 5 & 121 & 0.71457 & DW 12 & 2 & 179 & 0.71483 \\
\hline DUR 33 & 1 & 187 & 0.71056 & DW 12 & 3 & 197 & 0.71483 \\
\hline DUR 33 & 5 & 196 & 0.71037 & & & & \\
\hline
\end{tabular}

Table 3 (continued) 


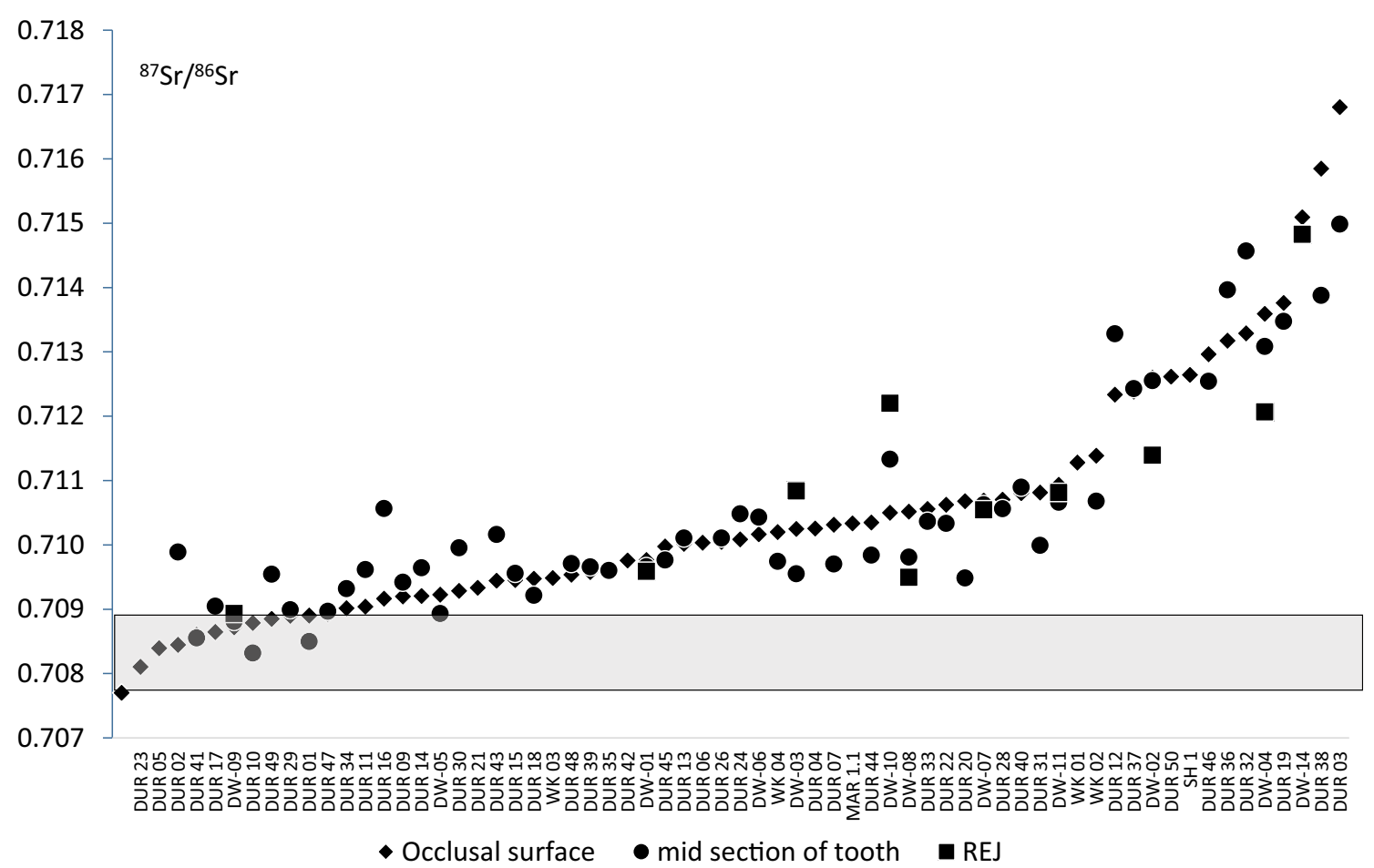

Fig. $4{ }^{87} \mathrm{Sr} /{ }^{86} \mathrm{Sr}$ variations within teeth. The data are arranged in increasing value of the occlusal (earliest developing) slice of each tooth and the field characteristic of chalk (local) values is marked as a shaded box

higher $\delta^{18} \mathrm{O}_{\text {carbvsmow values. This indicates that the animals }}$ from the Wessex sites are predominantly drawn from areas with higher drinking water values, i.e. those from more southern and western regions of Britain (Darling et al. 2003).

The strontium data further refines the origins of the cattle. Twenty percent of the animals are compatible with grazing on chalklands, which could infer an origin close to where they were found. The majority (66\%) of the Sr data fall between the upper limit of chalk 0.7089 and 0.7110 . These values are widely available in Britain and provide little geographical constraint. They could represent origins in the broader region of central southern Britain, but are also commonplace in various areas throughout the British Isles (Evans et al. 2018). However, the higher values between 0.711 and 0.713 are less common in the British biosphere. Fifteen samples from 11 individuals record values in this range. The closest areas to Durrington Walls and the other Late Neolithic sites that record this $\mathrm{Sr}$ isotope biosphere data range are in Wales and south west England. Similar values from cremated human remains were recently interpreted as likely to derive from West Wales (Snoeck et al. 2018). Such values are also recorded further north in Scotland. Eight animals (16 samples) record highly radiogenic Sr isotope compositions over 0.713 . According to current mapping (Evans et al. 2018), biosphere values of $>$ 0.7132 are virtually absent from southern Britain, though are present in restricted zones around the Malvern Hills (Chenery et al. 2010). They are recorded in small areas around the Lake District in northern England, but are most common across large areas of older geology in Scotland. However, there is growing evidence that, perhaps, there is a source of more radiogenic biosphere values available in England and Wales based on finds that seem unlikely, on archaeological grounds, to have originated in Scotland. Neolithic human data from Penywyrlod, South Wales (0.7132 to 0.7165$)$, have a very similar ${ }^{87} \mathrm{Sr} /{ }^{86} \mathrm{Sr}$ range to the most radiogenic Durrington Walls cattle. These individuals are interpreted as having spent their childhood in Wales (Neil et al. 2017). Similarly, Roman fauna from Caerleon, South Wales (Madgwick et al. 2019b), and Worcester (Gan et al. 2018) have produced highly radiogenic values, 0.71628 and 0.71582 respectively, suggesting that other radiogenic sources may be present in southern Britain.

An overseas origin is highly unlikely for the animals of this study, as the Late Neolithic is distinctive in having no evidence for continental contact (Vanderlinden 2012; Wilkin and Vanderlinden 2015) and therefore origins must be explored in a British context. Increasing evidence of pockets of radiogenic biosphere values may explain the origins of some of these animals. However, there can be little doubt that such values can only be attained in restricted areas of the landscape, and the large range of values $(0.7133$ to 0.7168$)$ in the dataset suggests that the animals in this study derive from different areas of radiogenic geology, potentially including Scotland.

In summary, very few of the animals could be described as locally raised with respect to a typical chalk-based $\mathrm{Sr}$ isotope value. However, large tracts of Britain can accommodate the 
Table 4 Mean values for ${ }^{87} \mathrm{Sr} /{ }^{86} \mathrm{Sr}$ isotope composition of cattle teeth

Sample

DUR 01

DUR 02

DUR 03

DUR 04

DUR 05

DUR 06

DUR 07

DUR 09

DUR 10

DUR 11

DUR 12

DUR 13

DUR 14

DUR 15

DUR 16

DUR 17

DUR 18

DUR 20

DUR 21

DUR 22

DUR 23

DUR 24

DUR 26

DUR 28

DUR 29

DUR 30

DUR 31

DUR 32

DUR 33

DUR 34

DUR 35

DUR 36

DUR 37

DUR 38

DUR 39

DUR 40

DUR 41

DUR 42

DUR 43

DUR 44

DUR 45

DUR 46

DUR 47

DUR 48

DUR 49

DW 01

DW 02

DW 03

DW 04
Table 4 (continued)

\begin{tabular}{lc}
\hline Sample & ${ }^{87} \mathrm{Sr}{ }^{86} \mathrm{Sr}$ \\
\hline DW 05 & 0.70908 \\
DW 06 & 0.71030 \\
DW 07 & 0.71062 \\
DW 08 & 0.70994 \\
DW 09 & 0.70882 \\
DW 10 & 0.71134 \\
DW 11 & 0.71080 \\
DW 12 & 0.71492 \\
SH 01 & 0.71264 \\
WK 01 & 0.71062 \\
WK 02 & 0.71139 \\
WK 03 & 0.71128 \\
WK 04 & 0.71020 \\
\hline
\end{tabular}

majority of the animals with Sr signatures between 0.7089 and 0.7110 , including areas within the broader region of central southern Britain. The oxygen isotope values suggest oxygen origins in western and southern Britain for most animals. The three samples with the lowest mean oxygen values (DUR 10, DUR 21, DUR 42) all have Sr isotope values between 0.708 and 0.710 and hence could therefore come from the central and eastern parts of Britain. Areas of southwest England and Wales could accommodate the origin of the animals with a tooth enamel strontium range between 0.711 and 0.713 and this would be supported by the oxygen data. The eight animals with values over 0.7132 cannot be excluded from a Scottish origin based on our current understanding of the British biosphere, but it is possible that some derive from unspecified areas of radiogenic geology in England and Wales.

\section{Comparison with pig and human data from Durrington Walls}

Durrington Walls was a major ceremonial site and, while this paper examines cattle data from there, a recent study provides a comparative dataset on pigs, which were the principal feasting animal at the site (Madgwick et al. 2019a).

Figure 6 highlights clear similarities in the range of values represented in both datasets, which can be divided into four groups based on breaks in the dataset shown by both pigs and cattle. The majority of animals (group 1) are consistent with an origin on the chalk and other Mesozoic deposits that dominate much of southern England and are common across Britain. Groups 2 and 3 are from more radiogenic terrains, probably characteristic of granitic and Palaeozoic areas, the closest of which are in southwest England and Wales (Evans et al. 2018). A final group (group 4) has distinctive values of $>$ 0.714 and must derive from areas of more radiogenic geology. As discussed, on the basis of current biosphere mapping data, 
Fig. 5 A box and whisker diagram comparing the average $\delta^{18} \mathrm{O}_{\text {carbvsmow values from }}$ animals in this study with the average values derived from central and eastern England data (Towers et al. 2011, 2014, 2017). The mean (x) and median (-) are given and the box represents the interquartile range (IQR) or central $50 \%$ of the data. The whiskers extend to beyond the IQR box by 1.5 times the IQR. Values beyond this range plot a dot

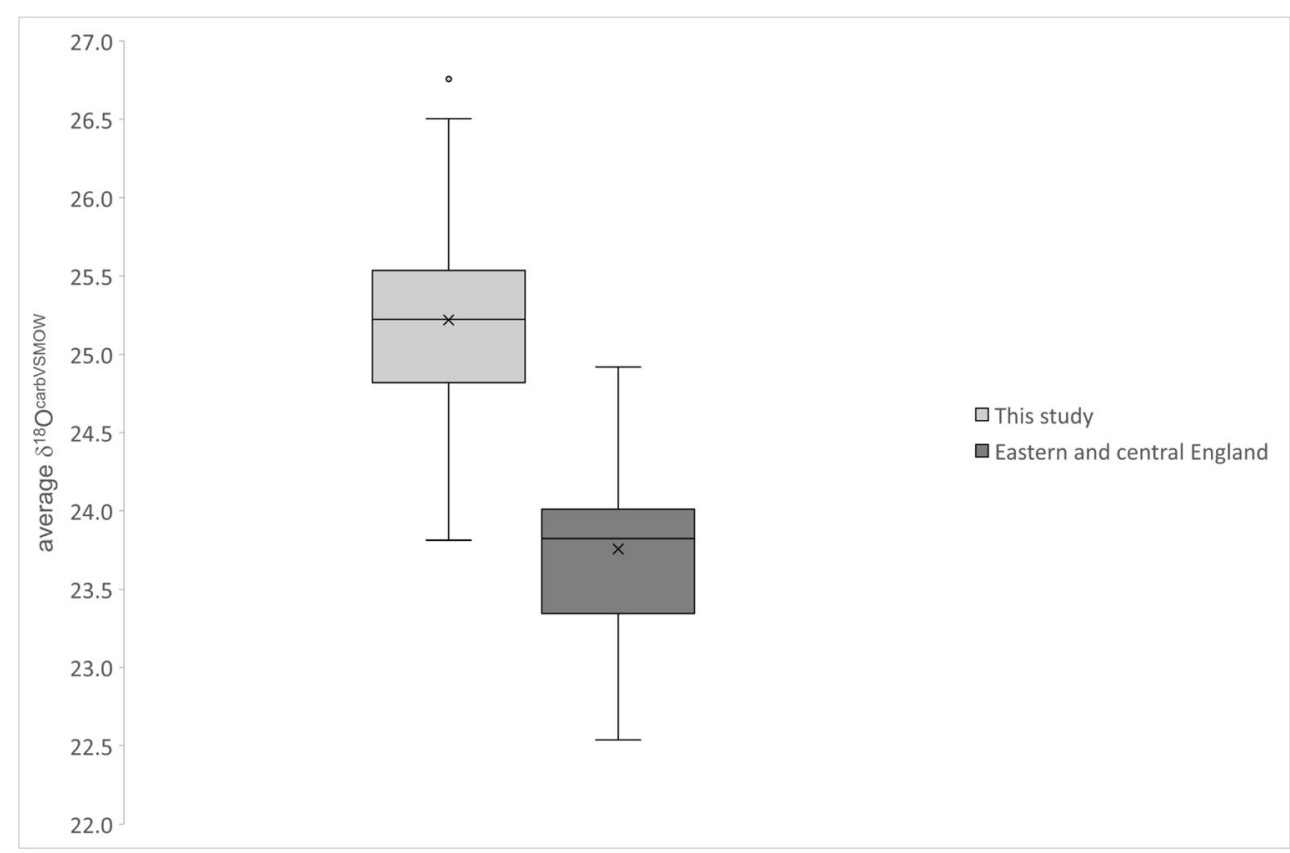

origins in Scotland cannot be excluded for at least some animals, but others may derive from radiogenic areas yet to be mapped in detail in Wales or England.

For both pigs and cattle, the majority of the data plot between 0.708 and 0.7108 . The datasets primarily differ in the proportion of animals with lower values associated with chalk: $28 \%$ of the pigs are below 0.709 , in contrast to $17 \%$ of the cattle. This suggests that a greater proportion of the pigs may have derived from chalk based terrain. However, this does not necessarily mean that the animals were raised on the Wessex chalkland surrounding Durrington Walls, as many of the pigs have an elevated sulphur value of $>14 \%$ o that indicates a coastal origin (Madgwick et al. 2019a). The dataset indicates that the people that raised and brought cattle and pigs to Durrington Walls and other Late Neolithic monumental centres came from wide-ranging areas, and that, in some instances, may have travelled with both types of livestock.

\section{The human tooth}

Human remains from Durrington Walls are very rare, and just one tooth (DUR 50) was available for isotope analysis. This tooth (an upper second premolar) produced an ${ }^{87} \mathrm{Sr} /{ }^{86} \mathrm{Sr}$ value of 0.7126 . This value is higher than values $(0.7078$ to 0.7118$)$ obtained from cremated bone from Stonehenge presented in Snoeck et al. (2018), but should not be treated as directly comparable. Cremated bone gives an average of strontium uptake in the years before death, rather than the more temporally defined value in childhood that the analysis of dental enamel provides. The result is inconsistent with the local chalk range and suggests that the individual moved to Durrington Walls sometime after the full mineralisation of the tooth (around age 6 years; Hillson 1996).

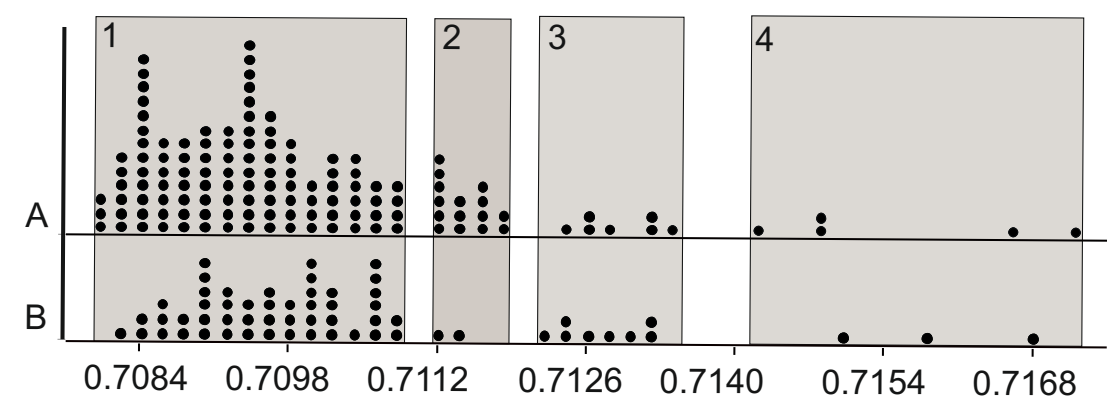

Fig. $6 \mathrm{~A}$ dot plot comparing the range and distribution of ${ }^{87} \mathrm{Sr} /{ }^{86} \mathrm{Sr}$ isotope composition in enamel from pig and cattle teeth from Late Neolithic feasting sites in southern England. The cattle are from Durrington Walls, West Kennet Palisade Enclosures, Stonehenge, and Marden and the pigs are from Durrington Walls, West Kennet Palisade Enclosures, Marden and Mount Pleasant. (A) Data from pigs taken from
Madgwick et al. (2019a), and (B) cattle data from this study. The data are grouped into four subsets based on breaks in distribution seen in both animal datasets. The pig data derives from a single sample from each individual and therefore average values of the cattle incremental samples are used for comparison 


\section{Conclusions}

These results have demonstrated the diversity of cattle origins at Late Neolithic Durrington Walls. The ${ }^{87} \mathrm{Sr} /{ }^{86} \mathrm{Sr}$ isotope data suggest that at least four distinct terrains are represented in the dataset. The majority of animals are consistent with an origin on the chalk and other Mesozoic deposits that dominate much of southern England and are common across Britain. Many of these animals must have been imported to the sites, though not necessarily over long distance. Two distinct groups of cattle are from more radiogenic terrains, probably characteristic of Palaeozoic areas, the closest of which are in southwest England and Wales. A final group has distinctive values of $>0.714$ and must derive from areas of even more radiogenic geology. On the basis of current biosphere mapping data, origins in Scotland seem likely for at least some animals, but others may derive from radiogenic areas incompletely mapped in England or Wales. Oxygen isotope data indicate that the majority of the animals are likely to derive from western or southern areas of Britain. Highland areas in the north of England and northeast Scotland are probably not represented in the dataset, but depleted oxygen isotope compositions suggest that some animals came from eastern and/or central areas of England.

Cattle are an important component of animal bone assemblages from Late Neolithic Britain. Remains of cattle were second only to pigs in abundance at Durrington Walls, and the presence of large quantities of cattle remains, along with evidence of butchery and burning on many bones, indicates that they were included in feasting activities (Albarella and Serjeantson 2002). The zooarchaeological evidence is also consistent with an introduction of cattle to Durrington Walls, due to the almost complete absence of neonatal bones. Such remains are expected to occur in breeding areas, because of natural casualties - their absence therefore suggests that husbandry largely occurred off-site.

The movement of cattle over long distances is an example of their importance in Neolithic society. Not only were they a significant source of food, but their role in feasting was important enough to warrant a huge investment of time and energy in herding them over long distances. These animals clearly had a role to play in sustaining long-distance networks in Late Neolithic Britain. As a proxy for human movement, the cattle from Durrington Walls are representative of the human journeys that were undertaken during the period and suggest links between human groups in many different parts of the country, both close and distant. The few cattle teeth from other contemporary sites hint that this phenomenon was more widespread and, perhaps, that Durrington Walls was not unique, but part of a wider network of connections and livestock exchange.
The exogenous origin of the livestock is in contrast with the largely local nature of the material culture (Chan et al. 2016). Animals could be driven on the hoof, while large quantities of objects would have been very onerous to carry. Such practical concern meant, however, that the local and the imported both played a role in the make-up of the Durrington Walls ceremonies, and probably contributed substantially to define the character of the communities occupying-permanently or periodically - the Stonehenge landscape.

Acknowledgements We are very grateful to Sarah Viner-Daniels who contributed substantially to the analysis of the data presented in this paper and the writing of an early draft. However, having moved to pastures new, she could not contribute to the latter stage of the project and therefore chose not be listed as a co-author. We are also grateful to all people (staff and students) who worked on site and contributed the collection of the remains discussed here. The animal remains discussed in this paper were collected as part of the Stonehenge Riverside Project and analysed as part of the Feeding Stonehenge Project, both funded by the Arts and Humanities Research Council (AHRC); without that support, this research would have not been possible. UA would like to acknowledge that he carried out some of the work leading to the preparation of this paper while on a British Academic mid-career fellowship, which provided him with research leave opportunity. We thank Dr. K Mee for providing the base map for Figure 1.

Open Access This article is distributed under the terms of the Creative Commons Attribution 4.0 International License (http:// creativecommons.org/licenses/by/4.0/), which permits unrestricted use, distribution, and reproduction in any medium, provided you give appropriate credit to the original author(s) and the source, provide a link to the Creative Commons license, and indicate if changes were made.

\section{References}

Albarella U, Payne S (2005) Neolithic pigs from Durrington Walls, Wiltshire, England: a biometrical database. J Archaeol Sci 32(4): 589-599

Albarella U, Serjeantson D (2002) A passion for pork: meat consumption at the British late Neolithic site of Durrington Walls. In: Miracle P, Milner N (eds) Consuming passions and patterns of consumption. Monographs of the McDonald Institute, Cambridge, pp 33-49

Balasse M (2002). Reconstructing dietary and environmental history from enamel isotopic analysis: Time resolution of intra-tooth sequential sampling. International Journal of Osteoarchaeology 12(3):155-165

Balasse M, Ambrose SH, Smith AB, Price TD (2002) The seasonal mobility model for prehistoric herders in the south-western cape of South Africa assessed by isotopic analysis of sheep tooth enamel. J Archaeol Sci 29(9):917-932

Balasse M, Tresset A, Dobney K, Ambrose SH (2005) The use of isotope ratios to test for seaweed eating in sheep. J Zool Soc Lond 266:283291

Balasse M, Tresset A, Ambrose SH (2006) Stable isotope evidence (delta $\mathrm{C}-13$, delta O-18) for winter feeding on seaweed by Neolithic sheep of Scotland. J Zool 270(1):170-176

Bayliss A, Cartwright C, Cook G, Griffiths S, Madgwick R, Marshall P, Reimer P (2017) Rings of fire and Grooved Ware settlement at West Kennet, Wiltshire. In: Bickle P, Cummings V, Hofmann D, Pollard J 
(eds) The Neolithic of Europe: essays in honour of Alasdair Whittle. Oxbow, Oxford, pp 249-278

Bendrey R, Hayes TE, Palmer MR (2009) Patterns of Iron Age horse supply: an analysis of strontium ratios in teeth. Archaeometry 51(1): $140-150$

Bentley RA (2006) Strontium isotopes from the earth to the archaeological skeleton: a review. J Archaeol Method Theory 13(3):135-187

Bentley RA, Knipper C (2005) Geographical patterns in biologically available strontium, carbon and oxygen isotope signatures in prehistoric SW Germany. Archaeometry 47(3):629-644

Birck JL (1986) Precision K-Rb-Sr isotopic analysis - application to RbSr chronology. Chem Geol 56(1-2):73-83

Bogaard A, Henton E, Evans JA, Twiss KC, Charles MP, Vaiglova P, Russell N (2013) Locating land use at Neolithic Çatalhöyük, Turkey: the implications of ${ }^{87} \mathrm{Sr} /{ }^{86} \mathrm{Sr}$ signatures in plants and sheep tooth sequences. Archeometry 56:860-877

Britton K, Grimes V, Dau J, Richards MP (2009) Reconstructing faunal migrations using intra-tooth sampling and strontium and oxygen isotope analyses: a case study of modern caribou (Rangifer tarandus granti). J Archaeol Sci 36(5):1163-1172

Britton KH, Grimes V, Niven L, Steele T, McPherron S, Soressi M, Kelly T, Jaubert J, Hublin J-J, Richards M (2011) Strontium isotope evidence for migration in late Pleistocene Rangifer: implications for Neanderthal hunting strategies at the Middle Palaeolithic site of Jonzac, France. J Hum Evol 61(2):176-185

Chan B, Viner S, Parker Pearson M, Albarella U, Ixer R (2016) Resourcing Stonehenge: patterns of human, animal and goods mobility in the late Neolithic. In: Leary J, Kador T (eds) Moving on in Neolithic Studies: understanding mobile lives. Oxbow Books, Oxford, pp 28-44

Chenery, C., G. Müldner, J. Evans, H. Eckardt and M. Lewis (2010). Strontium and stable isotope evidence for diet and mobility in Roman Gloucester, UK.Journal of Archaeological Science 37(1): $150-163$

Cleal RMJ, Walker KE, Montague R (1995) Stonehenge in its landscape: twentieth century excavations. English Heritage, London

Coplen TB (1988) Normalization of oxygen and hydrogen isotope data. Chem Geol 72:293-297

Darling WC, Bath AH, Talbot JC (2003). The O \& H stable isotopic composition of fresh waters in the British Isles: 2, Surface waters and groundwater. Hydrology and Earth System Sciences 7(2):183195

Ericson JE (1985) Strontium isotope characterization in the study of prehistoric human ecology. J Hum Evol 14:503-514

Evans J, Tatham S, Chenery S, Chenery C (2007) Anglo-Saxon animal husbandry techniques revealed through isotope and chemical variations in cattle teeth. Applied Geochem 22(9):1994-2005

Evans JA. Chenery CA, Mee K, Cartwright CE, Lee KA, Marchant AP, Hannaford L (2018) Biosphere Isotope Domains GB (V1): Interactive Website. British Geological Survey. (Interactive Resource). https://doi.org/10.5285/3b141dce-76fc-4c54-96fac232e98010ea

Gan YM, Towers J, Bradley RA, Pearson E, Nowell G, Peterkin J, Montgomery J (2018) Multi-isotope evidence for cattle droving at Roman Worcester. J Archaeol Sci Rep 20:6-17

Hare VJ, Loftus E, Jeffrey A, Ramsey CB (2018) Atmospheric CO2 effect on stable carbon isotope composition of terrestrial fossil archives. Nat Commun 9(1):252

Henton E, Meier-Augenstein W, Kemp HF (2010) The use of oxygen isotopes in sheep molars to investigate past herding practices at the Neolithic settlement of Çatalhöyük, Central Anatolia. Archaeometry 52(3):429-449

Hillson S (1996) Dental anthropology. Cambridge University Press, Cambridge
Hoppe KA, Koch PL, Carlson RW, Webb SD (1999) Tracking mammoths and mastodons: reconstruction of migratory behavior using strontium isotope ratios. Geology 27:439-442

Julien M, Bocherens H, Burke A, Drucker DG, Patou-Mathis M, Krotova $\mathrm{O}$, Péan S (2012) Were European steppe bison migratory? ${ }^{18} \mathrm{O},{ }^{13} \mathrm{C}$ and $\mathrm{Sr}$ intra-tooth isotopic variations applied to a palaeontological reconstruction. Quat Int 271:106-119

Kohn MJ (2010) Carbon isotope compositions of terrestrial C3 plants as indicators of (paleo) ecology and (paleo)climate. Proc Natl Acad Sci U S A 107(46):19691-19695

Leary J, Clarke A, Bell M (2016) Valley of the Henges: exploring Neolithic landscapes in the Pewsey Vale. Current Archaeology 316:28-34

Madgwick R, Mulville J (2015) Feasting on fore-limbs: conspicuous consumption and identity in later prehistoric Britain. Antiquity 89: 629-644

Madgwick R, Mulville J, Evans JA (2012) Investigating diagenesis and the suitability of porcine enamel for strontium $\left({ }^{87} \mathrm{Sr} /{ }^{86} \mathrm{Sr}\right)$ isotope analysis. J Anal At Spectrom 27:733-742

Madgwick R, Lamb AL, Sloane H, Nederbragt A, Albarella U, Parker Pearson M, Evans JA (2019a) Multi-isotope analysis reveals that feasts in the Stonehenge environs and across Wessex drew people and animals from throughout Britain. Sci Adv 5:eaau6078

Madgwick R, Lewis J, Grimes V, Guest P (2019b) On the hoof: exploring the supply of animals to the Roman legionary fortress at Caerleon using strontium $\left({ }^{87} \mathrm{Sr} /{ }^{86} \mathrm{Sr}\right)$ isotope analysis. Archaeol Anthropol Sci 11:223-235

Marshall P (In prep) Radiocarbon dating. In: Parker Pearson M, Pollard J, Richards C, Thomas J, Tilley C, Welham K (eds) Durrington Walls and Woodhenge: a place for the living. The Stonehenge Riverside Project volume 3. Sidestone Press, Leiden

Minniti C, Valenzuela-Lamas S, Evans J, Albarella U (2014) Widening the market. Strontium isotope analysis on cattle teeth from Owslebury (Hampshire, UK) highlights changes in livestock supply between the Iron Age and the Roman period. J Archaeol Sci 42: 305-314

Neil S, Montgomery J, Evans J, Cook GT, Scarre C (2017) Land use and mobility during the Neolithic in Wales explored using isotope analysis of tooth enamel. Am J Phys Anthropol 164(2):371-393

Parker Pearson M, Pollard J, Richards C, Thomas J, Welham K, Albarella U, Chan B, Marshall P, Viner S (2011) Feeding Stonehenge: feasting in late Neolithic Britain. In: Aranda Jiménez G, Montón-Subias S, Sánchez Romero M (eds) Guess who's coming to dinner: feasting rituals in the prehistoric societies of Europe and the Near East. GB, Oxbow Books, Oxford, pp 73-90

Pollard AM, Pellegrini M, Lee-Thorpe JA (2011). Technical Note: Some observations on the conversion of dental enamal $\mathrm{d} 18 \mathrm{O}$ values to d180W to determine human mobility. Americal Journal of Physical Anthropology 145(3):499-504

Richards C, Thomas J (1984) Ritual activity and structured deposition in Later Neolithic Wessex. In: Bradley R, Gardiner J (eds) British Archaeological Reports British Series 133Neolithic Studies: a review of some current research. BAR, Oxford, pp 189-218

Schwartz HP, Shoeninger MJ (1991) Stable isotope analyses in human nutritional ecology. Yearb Phys Anthropol 34:283-321

Snoeck C, Pouncett J, Claeys P, Goderis S, Mattielli N, Parker Pearson M, Willis C, Zazzo A, Lee-Thorp JA, Schulting RJ (2018) Strontium isotope analysis on cremated human remains from Stonehenge support links with west Wales. Sci Rep 8(1):10790

Sykes N, White J, Hayes T, Palmer M (2006) Tracking animals using strontium isotopes in teeth: the role of fallow deer (Dama dama) in Roman Britain. Antiquity. 80(310):948-959

Towers JJM, Evans J, Jay M, Parker Pearson M (2010) An investigation of the origins of cattle and aurochs deposited in the Early Bronze Age barrows at Gayhurst and Irthlingborough. J Archaeol Sci 37: 508-515 
Towers JMJ, Mainland I, Nehlich O, Montgomery J (2011) A calf for all seasons? The potential of stable isotope analysis to investigate prehistoric husbandry practices. J Archaeol Sci 38:1858-1868

Towers J, Gledhill A, Bond J, Montgomery J (2014) An investigation of cattle birth seasonality using delta $\mathrm{C}-13$ and delta $\mathrm{O}-18$ profiles within first molar enamel. Archaeometry 56:208-236

Towers J, Bond J, Evans J, Mainland I, Montgomery J (2017) An isotopic investigation into the origins and husbandry of Mid-Late Bronze Age cattle from Grimes Graves, Norfolk. J Archaeol Sci Rep 15: 59-72

Trickett MA, Budd P, Montgomery J, Evans J (2003) An assessment of solubility profiling as a decontamination procedure for the $\mathrm{Sr}-87 / \mathrm{Sr}-$ 86 analysis of archaeological human skeletal tissue. Appl Geochem 18(5):653-658

van der Jagt IMM, Kootker LM, van Kolfschoten T, Kars H, Davies GR (2012) An insight into animal exchange in Early Medieval Oegstgeest: a combined archaeozoological and isotopic approach. In: Raemaekers DCM, Esser E, Lauwerier RCGM, Zeiler JT (eds) A bouquet of archaeozoological studies: essays in honour of Wietske Prummel, pp 139-149

Vanderlinden M (2012) The importance of being insular. British Isles in the context of continental north-western Europe during the $3^{\text {rd }}$ millennium BC. In: Allen M, Gardiner J, Sheridan A (eds) Is there a British Chalcolithic? People, place and polity in the late $3^{\text {rd }}$ millennium BC. Oxbow, Oxford, pp 69-82
Viner S, Evans J, Albarella U, Parker Pearson M (2010) Cattle mobility in prehistoric Britain: strontium isotope analysis of cattle teeth from Durrington Walls (Wiltshire, Britain). J Archaeol Sci 37:2812-2820

Wainwright GJ, Longworth IH (1971) Durrington Walls: excavations 1966-1968. The Society of Antiquaries, London

Wainwright GJ, Evans JG, Longworth IH (1971) The excavation of a Late Neolithic Enclosure at Marden, Wiltshire. The Antiquaries Journal 51:177-239

Wilkin N, Vanderlinden M (2015) What was and what would never be: changing patterns of interaction and archaeological visibility across north-west Europe from 2,500 to 1,500 cal BC. In: AndersonWhymark H, Garrow D, Sturt F (eds) Continental connections: exploring cross-channel relationships from the Mesolithic to the Iron Age. Oxbow, Oxford, pp 99-121

Whittle, A. 1997. Sacred Mound, Holy Rings. Silbury Hill and the West Kennet palisade enclosures: a Later Neolithic complex in north Wiltshire. Oxbow Books: Oxford

Wright E, Viner-Daniels S, Parker Pearson M, Albarella U (2014) Age and season of pig slaughter at Late Neolithic Durrington Walls (Wiltshire, UK) as detected through a new system for recording tooth wear. J Archaeol Sci 52:497-514

Publisher's note Springer Nature remains neutral with regard to jurisdictional claims in published maps and institutional affiliations. 